\title{
The Dirichlet Problem for a Complex Monge-Ampère Equation
}

\author{
Eric Bedford ${ }^{1 \star}$ (New York) and B. A. Taylor ${ }^{2 \star \star}$ (Ann Arbor) \\ 1 Courant Institute of Mathematical Sciences, 251 Mercer, New York, NY 10012, USA \\ 2 Department of Mathematics, University of Michigan, Ann Arbor, MI 48104, USA
}

\section{Introduction}

On $\mathbb{C}^{n}$, write $d=\partial+\bar{\partial}$ and $d^{c}=i(\bar{\partial}-\partial)$ so that $d d^{c} u=2 i \partial \bar{\partial} u$, and let

$$
\beta_{n}=\left(\frac{i}{2}\right)^{n} \prod_{j=1}^{n} d z_{j} \wedge d \bar{z}_{j}
$$

be the usual volume form. We study here the nonlinear Dirichlet problem

$$
\begin{aligned}
\left(d d^{c} u\right)^{n}= & d d^{c} u \wedge \cdots \wedge d d^{c} u=f \beta_{n} \text { on } \Omega \\
& u \text { plurisubharmonic on } \Omega \\
& u=\phi \text { on } \partial \Omega,
\end{aligned}
$$

where $\Omega$ is a strictly pseudoconvex bounded open set in $\mathbb{C}^{n}$ and $f \geqq 0$. The operator $\left(d d^{c}\right)^{n}$ has an invariance property under holomorphic mappings, i.e. if $G=\left(g_{1}, \ldots, g_{n}\right)$ is analytic, $u \in C^{2}$, then

$$
\left(d d^{c}(u \circ G)\right)^{n}=\left|G^{\prime}\right|^{2 n}\left(d d^{c} u\right)^{n}
$$

where $G^{\prime}$ denotes the Jacobian determinant of $G$. Furthermore, if $G \neq(0, \ldots, 0)$, then $\left(d d^{c} \log |G|\right)^{n}=0$. Thus, for $f=0$, (1) is a natural generalization of the Dirichlet problem for harmonic functions in the complex plane.

Other extended Dirichlet problems were studied in connection with function theory in several variables by S. Bergman $[2,3]$ (on domains with distinguished boundary surfaces) and more generally by H. Bremermann [4]. In Section 8 it is shown that the solution of the problem discussed by Bremermann actually solves (1), in a generalized sense, with $f=0$. The problem (1) seems to be a reasonable candidate for a (nonlinear) potential theory associated with the theory of functions of several complex variables.

The question of uniqueness for the problem (1) is related to the question of existence of "inner functions" on the domain $\Omega$. If $h$ is a bounded analytic function

* Research supported in part by a Sloan Foundation Grant to Courant Institute of Mathematical Sciences, New York University and the Army Research Office grant number DAHCO4-75-G-0149 $\star \star$ Research supported in part by the National Science Foundation grant number GP37628 
on $\Omega$ such that $|h|=1$ almost everywhere on $\partial \Omega$, then the function $u=\log \frac{1}{2}\left(|h|^{2}+1\right)$ satisfies (1) with $n \geqq 2, f=0$ and $\phi=0$, and $u=\phi$ a.e. on $\partial \Omega$. Thus, if it were known that uniqueness held for (1) with $u \in C^{\infty}(\Omega) \cap L^{\infty}(\Omega)$ and boundary values taken almost everywhere, then one could conclude that $|h|$, and therefore $h$, must be constant.

Another possible application to complex analysis has been pointed out by Kerzman, Kohn, and Nirenberg [15]. They have shown that a regularity theorem for (1) would be sufficient to show that a proper holomorphic map between smooth, strictly pseudoconvex domains must have a smooth extension to the boundary. The regularity theorem desired is that a solution $u \in C(\bar{\Omega}) \cap C^{2}(\Omega)$ of (1) with $\phi=0, f^{1 / n} \in C_{0}^{\infty}(\Omega)$ must satisfy $u \in C^{\infty}(\bar{\Omega})$.

A closely related operator of Monge-Ampere type arises in the asymptotic behavior of the Bergman kernel function of a strongly pseudoconvex domain (see Hormander [14], Christoffers [8], and Diederich [9]). Also, Fefferman [10] has shown how this related Monge-Ampere operator is connected with the work of Chern and Moser [7] concerning analytic invariants of hypersurfaces in $\mathbb{C}^{n}$.

Since

$\left(d d^{c} u\right)^{n}=4^{n} n ! \operatorname{det}\left(\frac{\partial^{2} u}{\partial z_{j} \partial \bar{z}_{k}}\right) \beta_{n}$

the operator $\left(d d^{c}\right)^{n}$ appears to be a complex version of the Monge-Ampere determinant $\operatorname{det}\left(\frac{\partial^{2} u}{\partial x_{j} \partial x_{k}}\right)$. For example, when $n=2$ and $(z, w)$ are the variables in $\mathbb{C}^{2}$, the equation of (1) is

$$
u_{z \bar{z}} u_{w \bar{w}}-u_{z \bar{w}} u_{\bar{z} w}=f(z, w)
$$

while the real Monge-Ampere equation for $u=u(x, y)$ is

$$
u_{x x} u_{y y}-u_{x y}^{2}=f(x, y) \text {. }
$$

The real Monge-Ampere equations have been studied extensively in relation to problems in differential geometry, but it seems to be difficult to solve them in a completely satisfactory way. A.D. Alexandrov, using the theory of convex surfaces, showed the existence and uniqueness of convex (generalized) solutions to certain real Monge-Ampere equations (see [19] and the survey article by Gluck [11]). Interior regularity of the solution was discussed for $n \geqq 3$ by Pogorelov [19] and more generally by S. Y. Cheng and S.T. Yau [5].

A good geometric interpretation of the complex Monge-Ampere equation seems to be lacking, and the techniques used here for the complex case do not have the geometric flavor of the work for the real Monge-Ampere equation. In particular, there seem be several inequivalent ways of defining generalized solutions of (1). For an arbitrary plurisubharmonic function $u$, it is known that $d d^{c} u$ is a positive current of type $(1,1)([17]$, p. 70$)$, but it is not clear that the higher powers of $d d^{c} u$ are well-defined. In fact (in contrast to the real Monge-Ampere operator on convex functions) examples indicate that it is probably not possible to define $\left(d d^{c} u\right)^{n}$ as a distribution for all plurisubharmonic functions $u$ ([22]).

For the special case where $u$ is plurisubharmonic and $u \in C^{\infty}\left(\Omega \backslash\left\{z_{0}\right\}\right),\left(d d^{c} u\right)^{n}$ is well defined on $\Omega \backslash\left\{z_{0}\right\}$ and one may define the "mass" of $\left(d d^{c} u\right)^{n}$ at $z_{0}$ via 
integration by parts

$$
\left(d d^{c} u\right)^{n}\left\{z_{0}\right\}=\lim _{\varepsilon \rightarrow 0} \int_{\left|z-z_{0}\right|=\varepsilon} d^{c} u \wedge\left(d d^{c} u\right)^{n-1}
$$

provided the limit exists. This definition, while useful for many purposes, is in a sense too general for the Dirichlet problem (1), since uniqueness fails (see Example III of Section 4). A method for defining $\left(d d^{c} u\right)^{2}$ is provided via integration by parts (see Proposition 2.1),

$$
\int \phi \wedge\left(d d^{c} u\right)^{2}=-\int d d^{c} \phi \wedge d u \wedge d^{c} u
$$

which defines $\left(d d^{c} u\right)^{2}$ as a current of bidegree $(2,2)$ whenever $\nabla u$, the gradient of $u$, is locally square integrable. If $u$ is a bounded plurisubharmonic function, then $\boldsymbol{\nabla u}$ is locally square integrable, and consequently the formula (3) defines $\left(d d^{c} u\right)^{2}$ as a positive current of bidgree $(2,2)$.

For bounded, $C^{2}$ plurisubharmonic functions $u$ on an open set $\Omega$ in $\mathbb{C}^{n}$, Chern, Levine, and Nirenberg have given in [6] the estimate

$$
\int_{K}\left(d d^{c} u\right)^{n} \leqq C\left(\|u\|_{\Omega}\right)^{n}
$$

where $K$ is a compact subset of $\Omega$ and $\|u\|_{\Omega}=\sup \{|u(z)|: z \in \Omega\}$ With this estimate (and its proof), it is easy to show that the operator $u \rightarrow\left(d d^{c} u\right)^{n}$, thought of as a mapping from the $C^{2}$ plurisubharmonic functions on $\Omega$ to the space of nonnegative Borel measures on $\Omega$, has a continuous extension to the space of continuous plurisubharmonic functions. The following "minimum principle" is derived for this extension of $\left(d d^{c}\right)^{n}$.

Theorem A. Let $\Omega$ be a bounded open set in $\mathbb{C}^{n}$. If $u, v \in C(\bar{\Omega})$ are plurisubharmonic, and if $\left(d d^{c} u\right)^{n} \leqq\left(d d^{c} v\right)^{n}$, then

$$
\min \{u(z)-v(z): z \in \bar{\Omega}\}=\min \{u(z)-v(z): z \in \partial \Omega\} .
$$

An immediate consequence of the theorem is that continuous solutions of (1) are unique. Furthermore, this result shows that the idea of "subsolutions" is meaningful for the operator $\left(d d^{c}\right)^{n}$. The main goal of this paper is to prove existence of generalized solutions of (1). We are motivated by the familiar Perron method of taking the upper envelope of the family of subsolutions

$$
\mathscr{F}(\phi, f)=\left\{v \text { plurisubharmonic, } v \in C(\bar{\Omega}), v \leqq \phi \text { on } \partial \Omega,\left(d d^{c} v\right)^{n} \geqq f\right\}
$$

of (1). At this point, however, essential difficulties arise. For instance, it is not a priori clear whether $\left(d d^{c} u\right)^{n}$ can be defined where $u=\sup \{v: v \in \mathscr{F}\}$ is the upper envelope of subsolutions.

In order to avoid this difficulty another notion of generalized solution, introduced in Section 5, is considered. This is the operator $\Phi(u)$, which is essentially $\left(\operatorname{det}\left(u_{j k}\right)\right)^{1 / n} . \Phi$ is obtained from $d d^{c} u$ via a general measure-theoretic construction of Goffman and Serrin [12] and is thus defined for all plurisubharmonic $u$. Furthermore, $\Phi$ is well-behaved under convolution and weak limits. Thus, a more natural consideration is the Perron-Bremermann family

$$
\mathscr{B}(\phi, f)=\left\{v \text { plurisubharmonic, } \Phi(v) \geqq f, \lim _{\zeta \rightarrow z} \sup v(\zeta) \leqq \phi(z), z \in \partial \Omega\right\} \text {. }
$$


The following "maximum principle" for $\left(d d^{c}\right)^{n}$ is a consequence of the good behavior of $\Phi$ under convolution.

Theorem B. Suppose $u \in C^{2}(\vec{\Omega}), u$ is plurisubharmonic on $\Omega$, and $u$ solves (1) with $f^{1 / n}$ plurisubharmonic on $\Omega$. Then if $\left(a_{j k}\right)$ is a nonnegative Hermitian matrix,

$$
\begin{aligned}
\max & \left\{\sum \frac{\partial^{2} u(z)}{\partial z_{j} \partial \bar{z}_{k}} a_{j k}: z \in \Omega\right\} \\
& =\max \left\{\sum \frac{\partial^{2} u(z)}{\partial z_{j} \partial \bar{z}_{k}} a_{j k}: z \in \partial \Omega\right\} .
\end{aligned}
$$

From the "uppersemicontinuity" of $\Phi$, one may obtain the following regularity result, proved in Section 6.

Theorem C. Let $\Omega$ be the unit ball in $\mathbb{C}^{n}$, and let $\phi \in C^{2}(\partial \Omega), g \in C^{2}(\bar{\Omega}), g \geqq 0$. If $u=\sup \{v: v \in \mathscr{B}(\phi, g)\}$ is the upper envelope of $\mathscr{B}(\phi, g)$, then $u \in C(\bar{\Omega})$ and the second partial derivatives of $u$ exist almost everywhere on $\Omega$ and are locally bounded.

With this regularity theorem, it is possible to use real-variable arguments to show that the upper envelope $u$ of $\mathscr{B}(\phi, g)$ actually solves (1) with $f=g^{n}$ in the special case where $\Omega$ is the unit ball in $\mathbb{C}^{n}$. This allows us to make spherical modifications and obtain generalized solutions for more general domains.

Theorem D. Let $\Omega$ be a strictly pseudoconvex bounded domain in $\mathbb{C}^{n}$. If $\phi \in C(\partial \Omega)$, $f \in C(\bar{\Omega}), f \geqq 0$, then there exists a unique plurisubharmonic $u \in C(\bar{\Omega})$ such that

$$
\begin{aligned}
& \left(d d^{c} u\right)^{n}=f, \\
& \Phi(u)=f^{1 / n}
\end{aligned}
$$

and $u=\phi$ on $\partial \Omega$. Furthermore, if $\partial \Omega$ is smooth, $\phi \in C^{2}(\partial \Omega)$, and $f^{1 / n} \in \operatorname{Lip}^{1}(\bar{\Omega})$, then $u \in \operatorname{Lip}^{1}(\Omega)$.

The organization of the paper is as follows. In Section 2, we define the extension of $\left(d d^{c}\right)^{n}$ and give some of its properties. In Section 3 the minimum principle, Theorem A, is proved, and in Section 4 several examples, including nonuniqueness in the Dirichlet problem, are given. The operator $\Phi(u)=\left(\operatorname{det}\left(u_{j k}\right)\right)^{1 / n}$ is defined, and its properties are given in Section 5 . The results involving the family $\mathscr{B}$, including Theorems $B$ and $C$, are proved in Section 6. Section 7 contains some technical details concerning approximations to the Laplacian. The existence Theorem D is proved in Section 8. Finally, in Section 9, we discuss the relationship with Bremermann's Dirichlet problem, and apply the regularity result Theorem C to show some smoothness of parts of the boundary of certain envelopes of holomorphy.

\section{The Operator $\left(d d^{c} u\right)^{n}$}

For $\Omega$ an open set in $\mathbb{C}^{n}$, let $P(\Omega)$ denote the space of all plurisubharmonic functions on $\Omega$, and $C(\Omega), C^{k}(\Omega)$, etc. the usual spaces of continuous functions, $k$ times 

continuously differentiable functions, etc. If $u \in C^{2}(\Omega)$, let $\mathscr{H}(u)=\left[\frac{\partial^{2} u}{\partial z_{i} \partial \bar{z}_{j}}\right]$ denote
the complex Hessian of $u$. Then

$$
4^{n} n ! \operatorname{det} \mathscr{H}(u) \beta_{n}=\underbrace{d d^{c} u \wedge \cdots \wedge d d^{c} u}_{n \text { times }}=\left(d d^{c} u\right)^{n} .
$$

If $u$ is a plurisubharmonic function on $\Omega$, then the second partial derivatives $\frac{\partial^{2} u}{\partial z_{i} \partial \bar{z}_{j}}$ of $u$ are Borel measures on $\Omega$, and $d d^{c} u$ is a positive current of type $(1,1)$ on $\Omega([17]$, p. 70$)$. It is not clear, however, that the higher exterior powers of $d d^{c} u$ are well-defined, since their definition involves multiplication of the measure coefficients of $d d^{c} u$. In fact, examples indicate that it is probably not possible to define $\left(d d^{c} u\right)^{n}$ as a distribution for all plurisubharmonic functions ([22]). However, in [6], Chern, Levine, and Nirenberg have given the definition of $\left(d d^{c} u\right)^{n}$ for continuous plurisubharmonic functions $u$. Their method is to derive norm estimates for the positive measure $\left(d d^{c} u\right)^{n}$ when $u$ is smooth and then define $\left(d d^{c} u\right)^{n}$ for more general functions $u$ by approximation. We need some continuity properties of the operator in our argument, so in this section we will recall the arguments of [6] and note some of their consequences. In particular, we will prove an inequality for $\left(d d^{c} u\right)^{n}$, Theorem 2.4 , which shows this measure cannot be concentrated on an analytic subvariety when $u$ is continuous. We will use the following formulas (c.f. [6]).

Proposition 2.1. Let $1 \leqq m \leqq n$. Let $u_{1}, \ldots, u_{m} \in C^{2}(\Omega)$, and let $\phi$ be an differential form of type $(n-m, n-m)$ with coefficients from $C_{0}^{\infty}(\Omega)$. Then, for $m \geqq 2$,

$$
\int \phi \wedge d d^{c} u_{1} \wedge \cdots \wedge d d^{c} u_{m}=-\int d d^{c} \phi \wedge d u_{1} \wedge d^{c} u_{2} \wedge d d^{c} u_{3} \wedge \cdots \wedge d d^{c} u_{m}
$$

and

$$
\int \phi \wedge d d^{c} u_{1} \wedge \cdots \wedge d d^{c} u_{m}=\int u_{1} d d^{c} \phi \wedge d d^{c} u_{2} \wedge \cdots \wedge d d^{c} u_{m}
$$

Proof. Note that, by Stokes' theorem,

$$
\int \phi \wedge d d^{c} u_{1} \wedge \cdots \wedge d d^{c} u_{m}=-\int d \phi \wedge d^{c} u_{1} \wedge d d^{c} u_{2} \wedge \cdots \wedge d d^{c} u_{m}
$$

However, the $(n-(m-1), n-(m-1))$ parts of $d \phi \wedge d^{c} u$ and $d u \wedge d^{c} \phi$ are the same, so the last integral is equal to

$$
-\int d u_{1} \wedge d^{c} \phi \wedge d d^{c} u_{2} \wedge \cdots \wedge d d^{c} u_{m}=\int u_{1} d d^{c} \phi \wedge d d^{c} u_{2} \wedge \cdots \wedge d d^{c} u_{m}
$$

To prove (1), note also that

$$
\begin{aligned}
& -\int d u_{1} \wedge d^{c} \phi \wedge d d^{c} u_{2} \wedge \cdots \wedge d d^{c} u_{m}=-\int d^{c} u_{2} \wedge d\left[d u_{1} \wedge d^{c} \phi \wedge d d^{c} u_{3} \wedge \cdots \wedge d d^{c} u_{m}\right] \\
& =-\int d d^{c} \phi \wedge d u_{1} \wedge d^{c} u_{2} \wedge d d^{c} u_{3} \wedge \cdots \wedge d d^{c} u_{m}
\end{aligned}
$$

From the formulas of Proposition 2.1, we can read off some conditions under which $\left(d d^{c} u\right)^{n}$ should be defined. For example, when $m=n=2$, we have from (1) (with $u_{1}=u_{2}$ ),

$$
\int \phi\left(d d^{c} u\right)^{2}=-\int d u \wedge d^{c} u \wedge d d^{c} \phi
$$


Thus, when $\nabla u$, the gradient of $u$, is locally square integrable, this equation defines $\left(d d^{c} u\right)^{2}$ as a distribution of order at most 2. Similarly, from (2) we have

$$
\int \phi\left(d d^{c} u\right)^{2}=\int u d d^{c} \phi \wedge d d^{c} u
$$

which defines the left-hand side when $d d^{c} u$ has coefficients which are measures and $u$ is locally an $L^{1}$ function with respect to these measures. We will only consider plurisubharmonic functions here, and the situation when $\left(d d^{c} u\right)^{n}$ is a measure.

Denote by $M_{m}(\Omega)$ the class of currents on $\Omega$ of bidegree $(m, m)$ and order 0 . That is, $M_{m}(\Omega)$ is the class of differential forms of bidegree $(m, m)$ whose coefficients are Borel measures. We will suppose that $M_{m}(\Omega)$ has the usual topology of weak convergence in the space of measures. That is, $\mu_{j} \rightarrow \mu$ in $M_{m}(\Omega)$ if and only if $\int \phi \wedge \mu_{j} \rightarrow \int \phi \wedge \mu$ for all differential forms $\phi$ of bidegree $(n-m, n-m)$ with coefficients from $C_{0}(\Omega)$, the space of continuous functions with compact support in $\Omega$. For reference we record the following well-known fact.

Proposition 2.2. If $\mu_{j}, \mu \in M_{m}(\Omega)$, then $\mu_{j} \rightarrow \mu$ if and only if

(1) $\int \phi \wedge \mu_{j} \rightarrow \int \phi \wedge \mu$ for all differential forms $\phi$ of bidegree $(n-m, n-m)$ with coefficients from $C_{0}^{\infty}(\Omega)$; and

(2) for every compact subset $K$ of $\Omega$, there is a constant $C_{K}$ such that $\left\|\mu_{j}\right\|_{K} \leqq C_{K}$.

(Here, by $\|\mu\|_{K}$ we mean any total variation norm of $\mu$ on $K$. For example, if $\mu=\sum \mu_{I, J} d z^{I} \wedge d \bar{z}^{J}, \quad$ set $\|\mu\|_{K}=\sum\left\|\mu_{I, J}\right\|_{K}$

where $\left\|\mu_{I, J}\right\|_{K}$ is the usual total variation of the measure $\mu_{I, J}$ on the compact subset $K$ of $\Omega$.)

Using Proposition 2.1, it is now easy to see that $\left(d d^{c} u\right)^{n}$ is defined for all continuous plurisubharmonic functions $u$.

Proposition 2.3. For $1 \leqq m \leqq n$, consider $T_{m}(u)=\left(\left(d d^{c} u\right),\left(d d^{c} u\right)^{2}, \ldots,\left(d d^{c} u\right)^{m}\right), \quad a$ densely defined operator from $C(\Omega)$ to $\prod_{k=1}^{m} M_{k}(\Omega)$ with domain $C^{2}(\Omega)$. Then

(1) $T_{m}$ maps $C(\Omega)$-bounded subsets of $C^{2}(\Omega) \cap P(\Omega)$ into bounded subsets of $\prod_{k=1}^{m} M_{k}(\Omega) ;$ and

(2) If $u_{j}, v_{j} \in C^{2}(\Omega)$, and $\lim _{j \rightarrow \infty} u_{j}=\lim _{j \rightarrow \infty} v_{j}=u$ in $C(\Omega)$, and if both the limits $\lim _{j \rightarrow \infty} T_{m} v_{j}$ and $\lim _{j \rightarrow \infty} T_{m} v_{j}$ exist, then they are equal. Consequently, $T_{m}$ has an unique extension to a continuous operator on all of $C(\Omega) \cap P(\Omega)$.

Proof. The assertion (1) is a special case of a lemma of Chern, Levine, and Nirenberg ([6], p. 125).

To prove (2), let $u_{j}, v_{j}$ be as in the hypothesis. For $m=1$, the assertion is wellknown. It also follows from (1) of Proposition 2.1. Proceeding by induction, assume the assertion has been proved for smaller values of $m>1$. Then we know that $\lim _{j \rightarrow \infty}\left(d d^{c} u_{j}\right)^{k}=\lim _{j \rightarrow \infty}\left(d d^{c} v_{j}\right)^{k}=\mu_{k}$ for $k<m$. Let $\mu_{m}=\lim _{j \rightarrow \infty}\left(d d^{c} u_{j}\right)^{m}$ and $v_{m}=$ 
$\lim _{j \rightarrow \infty}\left(d d^{c} v_{j}\right)^{m}$. We have to show that $\mu_{m}=v_{m}$. That is, $\int \phi \wedge \mu_{m}=\int \phi \wedge v_{m}$ for all forms $\phi$ of type $(n-m, n-m)$ with coefficients from $C_{0}^{\infty}(\Omega)$.

Now, by (2) of Proposition 2.1, we have

$$
\int \phi \wedge \mu_{m}=\lim _{j \rightarrow \infty} \int \phi \wedge\left(d d^{c} u_{j}\right)^{m}=\lim _{j \rightarrow \infty} \int u_{j} d d^{c} \phi \wedge\left(d d^{c} u_{j}\right)^{m-1}
$$

and

$$
\int \phi \wedge v_{m}=\lim _{j \rightarrow \infty} \int v_{j} d d^{c} \phi \wedge\left(d d^{c} v_{j}\right)^{m-1}
$$

By the induction hypotheses, $\left(d d^{c} u_{j}\right)^{m-1}$ and $\left(d d^{c} v_{j}\right)^{m-1}$ both converge to $\mu_{m-1}$ in $M_{m-1}(\Omega)$. Also, $u_{j}, v_{j}$ converge uniformly to $u$ on support $\phi$. Thus, both limits are equal to $\int u d d^{c} \phi \wedge \mu_{m-1}$, as asserted. This completes the proof.

It is convenient to have a slightly better estimate for $\left(d d^{c} u\right)^{n}$ than the one given in [6]. We give one in the next theorem whose proof, however, follows closely the one given in [6].

Theorem 2.4. Let $u$ be plurisubharmonic and continuous on the closure of the polydisc

$$
\Delta\left(\lambda^{n} r\right)=\left\{\left(z_{1}, \ldots, z_{n}\right):\left|z_{j}\right|<\lambda^{n} r_{j}, 1 \leqq j \leqq n\right\},
$$

for some $\lambda>1$. Let $\omega(\delta)$ be the modulus of continuity of $u$ on $\Delta\left(\lambda^{n} r\right)$; i.e.

$$
|u(z)-u(\zeta)| \leqq \omega(\delta) \text { if }|z-\zeta| \leqq \delta .
$$

Then there is a constant $C$, depending only on $n$ and $\lambda>1$ such that

$$
\int_{\Delta(r)}\left(d d^{c} u\right)^{n} \leqq C \omega\left(r_{1}\right) \ldots \omega\left(r_{n}\right)
$$

Corollary 2.5. If $u$ is a continuous plurisubharmonic function on $\Omega \subset \mathbb{C}^{n}$, then $\left(d d^{c} u\right)^{n}$ has no mass concentrated on any analytic subvariety of $\Omega$.

Proof. Clearly, from Theorem $2.4,\left(d d^{c} u\right)^{n}$ has no mass at points. Let $V$ be a subvariety of dimension $k<n$. The singular points of $V$ are a subvariety of dimension $<k$, which we can assume has $\left(d d^{c} u\right)^{n}$ measure zero by induction. Thus, we only have to show that $\left(d d^{c} u\right)^{n}$ assigns measure zero to the regular points of $V$. This is a purely local question, so we can assume that $V=\left\{\left(z_{1}, \ldots, z_{k}, 0, \ldots, 0\right)\right\}$, near $0 \in \mathbb{C}^{n}$, and then the result follows by letting $r_{k+1} \rightarrow 0$ in the estimate of Theorem 2.4.

Remark. The estimate of Theorem 2.4 cannot be substantially improved. For example, in $\mathbb{C}^{2}$, if $u\left(z_{1}, z_{2}\right)=\max \left(0, \operatorname{Re} z_{1}, \operatorname{Re} z_{2}\right)$, then $\omega(\delta)$ is $\delta$, and it can be calculated that $\left(d d^{c} u\right)^{2}$ is equal to the Lebesgue surface area measure on the 2-dimensional real subspace $\operatorname{Re} z_{1}=\operatorname{Re} z_{2}=0$. Thus,

$$
\int_{\Delta\left(r_{1}, r_{2}\right)}\left(d d^{c} u\right)^{2}=4 r_{1} r_{2}
$$

We will obtain Theorem 2.4 as a special case of the following (slightly) more general statement which will be proved by induction.

Proposition 2.6. Let $u_{1}, \ldots, u_{n}$ be $C^{2}$ plurisubharmonic functions on the closure of $\Delta\left(\lambda^{n} r\right)$, with

$$
\left|u_{i}(z)-u_{i}(\zeta)\right| \leqq \omega(\delta) \text { for }|z-\zeta| \leqq \delta \text {, and } z, \zeta \in \Delta\left(\lambda^{n} r\right)
$$


There is a constant $C$, depending only on $n$ and $\lambda>1$ such that

$$
\int_{\Delta(r)} d d^{c} u_{1} \wedge \cdots \wedge d d^{c} u_{n} \leqq C \omega\left(r_{1}\right) \ldots \omega\left(r_{n}\right) .
$$

Proof. We will prove the Proposition by induction on $n$. The case $n=1$ is an immediate consequence of Jensen's formula. For if we set $n(r)=\frac{1}{2 \pi} \int_{|z|<r} d d^{c} u$, then
Jensen's formula is

$$
\frac{1}{2 \pi} \int_{-\pi}^{\pi} u\left(r e^{i \theta}\right) d \theta-u(0)=\int_{0}^{r} \frac{n(t)}{t} d t
$$

The left-hand side is clearly dominated by $\omega(r)$. For the right-hand side, the standard argument

$$
\int_{0}^{r} \frac{n(t)}{t} d t \geqq \int_{r / \lambda}^{r} \geqq n(r / \lambda) \log \lambda
$$

gives us an upper bound for $n(r)$ in terms $\omega(\lambda r)$, as asserted.

Now assume the Proposition has been proved for smaller values of $n$. Given $n-1$ functions $v_{1}, \ldots, v_{n-1}$, define the coefficients $\Delta(i, j)$ by

$$
d d^{c} v_{1} \wedge \cdots \wedge d d^{c} v_{n-1}=\sum_{i, j=1}^{n} \Delta(i, j) *\left(d z_{i} \wedge d \bar{z}_{j}\right)
$$

where $\left(\frac{\sqrt{-1}}{2}\right) d z_{i} \wedge d \bar{z}_{j} \wedge *\left(d z_{i} \wedge d \bar{z}_{j}\right)=\beta_{n}$. Also choose a $C^{\infty}$ function $\chi$ of one variable $\zeta \in \mathbb{C}$ such that $\chi(\zeta)=1$ if $|\zeta| \leqq 1, \chi(\zeta)=0$ if $|\zeta| \geqq \lambda$, and $0 \leqq \chi \leqq 1$. Set

$$
\phi\left(z_{1}, \ldots, z_{n}\right)=\prod_{j=1}^{n} \chi\left(\frac{z_{j}}{r_{j}}\right) .
$$

Since $u_{1}, \ldots, u_{n}$ are plurisubharmonic, $d d^{c} u_{1} \wedge \cdots \wedge d d^{c} u_{n}$ is a nonnegative measure $([17, \mathrm{p} .68)$ so

$$
\int_{\Delta(r)} d d^{c} u_{1} \wedge \cdots \wedge d d^{c} u_{n} \leqq \int \phi d d^{c} u_{1} \wedge \cdots \wedge d d^{c} u_{n}=I .
$$

We will estimate $I$.

It is no loss of generality to assume that $r_{1} \leqq r_{j}, 2 \leqq j \leqq n$. Define

$$
\tilde{u}_{i}\left(z_{1}, z_{2}, \ldots, z_{n}\right)=u_{i}\left(0, z_{2}, \ldots, z_{n}\right) \text {. }
$$

Writing $u_{1}=u_{1}-\tilde{u}_{1}+\tilde{u}_{1}$ in the expression for $I$, we have

$$
\begin{aligned}
I & =\int \phi d d^{c}\left(u_{1}-\tilde{u}_{1}\right) \wedge d d^{c} u_{2} \wedge \cdots \wedge d d^{c} u_{n}+\int \phi d d^{c} \tilde{u}_{1} \wedge d d^{c} u_{2} \wedge \cdots \wedge d d^{c} u_{n} \\
& =I_{1}+I_{2} .
\end{aligned}
$$

By (2) of Proposition 2.1, we have

$$
I_{1}=\int\left(u_{1}-\tilde{u}_{1}\right) d d^{c} \phi \wedge d d^{c} u_{2} \wedge \cdots \wedge d d^{c} u_{n}
$$

so

$$
I_{1} \leqq \omega\left(r_{1}\right) \int\left|d d^{c} \phi \wedge d d^{c} u_{2} \wedge \cdots \wedge d d^{c} u_{n}\right|
$$


With the notation of (2.3) and (2.4), we see that the coefficients in $d d^{c} \phi \wedge d d^{c} u_{2} \ldots d d^{c} u_{n}$ are linear combinations of the two kinds of terms:

$$
\left[\prod_{k \neq i, j} \chi\left(\frac{z_{k}}{r_{k}}\right)\right] \cdot \frac{\partial \chi}{\partial \zeta}\left(\frac{z_{i}}{r_{i}}\right) \frac{\partial \chi}{\partial \bar{\zeta}}\left(\frac{z_{j}}{r_{j}}\right) \frac{1}{r_{i} r_{j}} \Delta(i, j), i \neq j
$$

and

$$
\left[\prod_{k \neq i} \chi\left(\frac{z_{k}}{r_{k}}\right)\right] \frac{\partial^{2} \chi}{\partial \zeta \partial \bar{\zeta}}\left(\frac{z_{i}}{r_{i}}\right) \frac{1}{r_{i}^{2}} \Delta(i, i), \quad i=j
$$

Because all the forms $d d^{c} u_{2}, \ldots, d d^{c} u_{n}$ are nonnegative, the $n \times n$ matrix $[\Delta(i, j)]$ is a nonnegative Hermitian matrix ([17], p. 68). Consequently, $2\left(r_{i} r_{j}\right)^{-1}|\Delta(i, j)| \leqq$ $r_{i}^{-2} \Delta(i, i)+r_{j}^{-2} \Delta(j, j)$ and so for a suitable constant $C=C(n, \lambda)$, we have

$$
I_{1} \leqq C \omega\left(\lambda r_{1}\right) \sum_{i} \frac{1}{r_{i}^{2}} \int_{\Delta(\lambda r)} \Delta(i, i) d m(z)
$$

where $d m$ is Lebesgue measure. To estimate the last integrals, we will use the induction hypothesis. To be specific, suppose $i=1$. Let $\Delta_{1}=\left\{z_{1}:\left|z_{1}\right|<\lambda r_{1}\right\}$, $\Delta_{2}=\left\{\left(z_{2}, \ldots, z_{n}\right):\left|z_{j}\right|<\lambda r_{j}\right\}$. Then

$$
\int_{\Delta(\lambda r)} \Delta(i, i) d m(z)=\int_{\Delta_{1}} \int_{\Delta_{2}} \Delta(i, i) d m\left(z_{2}, \ldots, z_{n}\right) d m\left(z_{1}\right) .
$$

With $z_{1}$ fixed, the $n-1$ functions

$$
\left(z_{2}, \ldots, z_{n}\right) \rightarrow u_{j}\left(z_{1}, z_{2}, \ldots, z_{n}\right), \quad 2 \leqq j \leqq n
$$

all satisfy the hypotheses of the Proposition with $r$ replaced by $\lambda r$ and $n$ by $n-1$. Thus by induction, we have for some constant $C$

$$
\int_{\Delta(\lambda r)} \Delta(1,1) d m(z) \leqq C \omega\left(r_{2}\right) \ldots \omega\left(r_{n}\right) \cdot\left(r_{1}\right)^{2} .
$$

Consequently, for some (different) $C>0$, from (2.7) there follows

$$
I_{1} \leqq C \omega\left(\lambda r_{1}\right) \sum_{i=1}^{n} \prod_{j \neq 1} \omega\left(r_{j}\right)
$$

Now, it is no loss of generality to assume that $\omega$ is increasing and subadditive, because it can be replaced by the maximum of the modulus of continuity of the $u_{i}$. Hence $\omega\left(\lambda r_{1}\right)<2 \lambda \omega\left(r_{1}\right)$ for $\lambda \geqq 1$ and $\omega\left(r_{1}\right) \prod_{j \neq 1} \omega\left(r_{j}\right) \leqq \omega\left(r_{i}\right) \prod_{j \neq 1} \omega\left(r_{j}\right)$ because $r_{1} \leqq r_{i}$
$2 \leqq i \leqq n$. Thus

$$
I_{1} \leqq C \omega\left(r_{1}\right) \ldots \omega\left(r_{n}\right)
$$

for a suitable constant $C$.

To estimate the second integral $I_{2}$, we write $u_{2}=u_{2}-\tilde{u}_{2}+\tilde{u}_{2}$ so

$$
\begin{aligned}
I_{2}= & \int \phi d d^{c}\left(u_{2}-\tilde{u}_{2}\right) \wedge d d^{c} \tilde{u}_{1} \wedge d d^{c} u_{3} \wedge \cdots \wedge d d^{c} u_{n} \\
& +\int \phi d d^{c} \tilde{u}_{1} \wedge d d^{c} \tilde{u}_{2} \wedge d d^{c} u_{3} \wedge \cdots \wedge d d^{c} u_{n} \\
= & J_{1}+J_{2} .
\end{aligned}
$$


The first integral $J_{1}$ is estimated exactly as $I_{1}$ was above. If we repeat this process on $J_{2}$, we finally arrive at

$$
\begin{aligned}
& \int \phi d d^{c} \tilde{u}_{1} \wedge \cdots \wedge d d^{c} \tilde{u}_{n-1} \wedge d d^{c} u_{n} \\
& =\int \phi d d^{c}\left(u_{n}-\tilde{u}_{n}\right) \wedge d d^{c} \tilde{u}_{1} \wedge \cdots \wedge d d^{c} \tilde{u}_{n-1} \\
& \quad+\int \phi d d^{c} \tilde{u}_{1} \wedge \cdots \wedge d d^{c} \tilde{u}_{n} .
\end{aligned}
$$

Again the first integral can be handled as $I_{1}$ was, while the last integral vanishes because $\tilde{u}_{1}, \ldots, \tilde{u}_{n}$ are functions only of $n-1$ variables. This completes the proof.

Remark. The estimate of Theorem 2.4 can be slightly improved.An examination of the proof will show that $\omega\left(r_{1}\right) \ldots \omega\left(r_{n}\right)$ can be replaced by

$$
\omega_{1}\left(r_{1}\right) \ldots \omega_{n}\left(r_{n}\right)
$$

where each $\omega_{i}$ is the "modulus of continuity in the $i$-th variable." That is, for example,

$$
\omega_{1}(\delta)=\sup \left\{\left|u\left(z_{1}, z_{2}, \ldots, z_{n}\right)-u\left(\zeta, z_{2}, \ldots, z_{m}\right)\right|:\left|z_{1}-\zeta\right| \leqq \delta,\left|z_{j}\right| \leqq \lambda^{n} r_{j}\right\} .
$$

We next verify that the definition of $\left(d d^{c} u\right)^{n}$ in the weak sense coincides with the classical definition when it exists.

Proposition 2.7. Let $u \in C(\Omega) \cap P(\Omega)$ and suppose $d d^{c} u=2 i \sum h_{j k} d z_{j} \wedge d \bar{z}_{k}$, where the $h_{j k}$ are locally $L^{m}$ functions in $\Omega, 1 \leqq m \leqq n$. Then $\left(d d^{c} u\right)^{m} \in M_{m}(\Omega)$ has locally integrable coefficients and is given by $\left[2 i \sum_{j, k} h_{j k} d z_{j} \wedge d \bar{z}_{k}\right]^{m}$.

Proof. If $u$ satisfies the hypotheses of the proposition, then we can choose a sequence $u_{\varepsilon}$ of smooth plurisubharmonic functions converging to $u$ in $C(\Omega)$ such that $\frac{\partial^{2} u_{\varepsilon}}{\partial z_{j} \partial \bar{z}_{k}}$ converges to $h_{j \bar{k}}=\frac{\partial^{2} u}{\partial z_{j} \partial \bar{z}_{k}}$ locally in $L^{m}$. For example, $u_{\varepsilon}=u * \chi_{\varepsilon}$, where $\chi_{\varepsilon}$ is a usual smoothing kernel ([13], p. 45). The result now follows from the weak continuity of $\left(d d^{c} u\right)^{n}$, given by Proposition 2.3 .

Let us now prove two inequalities which are useful in the study of the Dirichlet problem.

Proposition 2.8. If $u, v \in C(\Omega) \cap P(\Omega)$, then

(1) $\quad\left(d d^{c}[\max (u, v)]\right)^{n} \geqq \min \left[\left(d d^{c} u\right)^{n},\left(d d^{c} v\right)^{n}\right]$.

(2) $\left(d d^{c}[u+v]\right)^{n} \geqq\left(d d^{c} u\right)^{n}+\left(d d^{c} v\right)^{n}$.

Proof. Let $\mu=\min \left[\left(d d^{c} u\right)^{n},\left(d d^{c} v\right)^{n}\right]$, and first assume that $\mu(\{z: u(z)=v(z)\})=0$. Then set $\Omega_{1}=\{u<v\}$, and $\Omega_{2}=\{v<u\}$. We have on $\Omega_{1},\left(d d^{c}[\max (u, v)]\right)^{n}=$ $\left(d d^{c} v\right)^{n} \geqq \mu$, and a similar inequality on $\Omega_{2}$. Since $\mu\left(\Omega \backslash\left(\Omega_{1} \cup \Omega_{2}\right)\right)=0$, and $\left(d d^{c}[\max (u, v)]\right)^{n} \geqq 0$, the assertion (1) follows in this case.

In general, replace $v$ by $v+\varepsilon, \varepsilon$ a small constant. Then $\max (u, v+\varepsilon) \rightarrow \max (u, v)$ in $C(\Omega)$ as $\varepsilon \rightarrow 0$. Thus, $\left(d d^{c}[\max (u, v+\varepsilon)]\right)^{n} \rightarrow\left(d d^{c}[\max (u, v)]\right)^{n}$ weakly, by Proposition 2.3. Also, since $\left(d d^{c} v\right)^{n}=\left(d d^{c}(v+\varepsilon)\right)^{n}$, we have

$$
\mu=\min \left[\left(d d^{c} u\right)^{n},\left(d d^{c}(v+\varepsilon)\right)^{n}\right] .
$$

Thus, by the first case, we know the inequality for $\max (u, v+\varepsilon)$ for all $\varepsilon$ with $\mu\{z: u(z)=v(z)+\varepsilon\}=0$. Since this happens for all but at most countably many $\varepsilon>0$, we obtain (1) letting $\varepsilon \rightarrow 0$, avoiding these countably many values of $\varepsilon$. 
Remark. Example II, Section 4 shows that (1) fails when $u, v$ are not continuous.

To prove (2), note first that if $u, v \in C^{2}(\Omega) \cap P(\Omega)$, the differential $(1,1)$ forms $d d^{c} u, d d^{c} v$ are positive and therefore so are sums of positive multiples of powers of the forms $([17]$, p. 68). Thus,

$$
\left(d d^{c}[u+v]\right)^{n}=\left(d d^{c} u\right)^{n}+\left(d d^{c} v\right)^{n}+\sum_{j=1}^{n-1}\left(\begin{array}{l}
n \\
j
\end{array}\right)\left(d d^{c} u\right)^{j} \wedge\left(d d^{c} v\right)^{n-j} \geqq\left(d d^{c} u\right)^{n}+\left(d d^{c} v\right)^{n} .
$$

If $u, v$ are only continuous, let $u_{\varepsilon}=u * \chi_{\varepsilon}, v_{\varepsilon}=v * \chi_{\varepsilon}$ where $\chi_{\varepsilon}$ is a usual smoothing kernel $\left([13]\right.$, p. 45). Since $u_{\varepsilon}, v_{\varepsilon}$ are smooth, we have $\left(d d^{c}\left[u_{\varepsilon}+v_{\varepsilon}\right]\right)^{n} \geqq\left[d d^{c} u_{\varepsilon}\right]^{n}$ $+\left[d d^{c} v_{\varepsilon}\right]^{n}$. However, $u_{\varepsilon} \rightarrow u, v_{\varepsilon} \rightarrow v$ and $u_{\varepsilon}+v_{\varepsilon} \rightarrow u+v$ in $C(\Omega)$, so by Proposition 2.3, we obtain (2) by letting $\varepsilon \rightarrow 0$.

The question arises whether there is a "natural" domain of definition for $\left(d d^{c} u\right)^{n}$. As was pointed out following Proposition 1, if $\nabla u$ is locally in $L^{2}$, then $\left(d d^{c} u\right)^{2}$ is a $(2,2)$-current. Is there an analogue of this for $n>2$ ? It is not hard to show that a bounded subharmonic function has locally square integrable gradient, and thus $\left(d d^{c} u\right)^{2}$ is a positive (2,2)-current for any bounded plurisubharmonic function.

Proposition 2.9. If $u \in L^{\infty}(\Omega) \cap P(\Omega)$ one may define $\left(d d^{c} u\right)^{k}, 1 \leqq k \leqq n$ inductively as follows. If $\chi$ is a $(n-k, n-k)$-form with smooth coefficients with compact support in $\Omega$, then

$$
\int\left(d d^{c} u\right)^{k} \wedge \chi=\int u\left(d d^{c} u\right)^{k-1} \wedge d d^{c} \chi .
$$

Defined in this manner, $\left(d d^{c} u\right)^{k}$ is a positive $(k, k)$-current.

Proof. The proposition is true for $k=1$ so we proceed by induction.

Since $u$ is uppersemicontinuous and bounded, $u\left(d d^{c} u\right)^{k-1}$ again has measure coefficients, and thus $\left(d d^{c} u\right)^{k}$ is a $(k, k)$-current. Positivity can be seen as follows. Let $\chi$ be a smooth $(n-k, n-k)$-form with compact support taking values in the positive cone $\mathscr{P}$ (see Lelong [17], p. 60). It must be shown that

$$
\int\left(d d^{c} u\right)^{k} \wedge \chi \geqq 0 .
$$

Since $u$ is plurisubharmonic, there is a sequence of functions $u_{j} \in P(\Omega) \cap C^{\infty}(\Omega)$ decreasing monotonically to $u$. Again $d d^{c} u_{j} \wedge \chi$ takes values in $\mathscr{P}$, and thus by induction

$$
\int\left(d d^{c} u\right)^{k-1} \wedge\left(d d^{c} u_{j} \wedge \chi\right) \geqq 0
$$

The positivity of $\left(d d^{c} u\right)^{k}$ now follows by monotone convergence,

$$
\begin{aligned}
\int\left(d d^{c} u\right)^{k} \wedge \chi & =\int u\left(d d^{c} u\right)^{k-1} \wedge d d^{c} \chi \\
& =\lim _{j \rightarrow \infty} \int u_{j}\left(d d^{c} u\right)^{k-1} \wedge d d^{c} \chi \\
& =\lim _{j \rightarrow \infty} \int\left(d d^{c} u\right)^{k-1} \wedge d d^{c} u_{j} \wedge \chi \geqq 0 .
\end{aligned}
$$

The difficulty with this definition is that it is not clear whether there is a topology on $L^{\infty}(\Omega) \cap P(\Omega)$ for which $C(\Omega) \cap P(\Omega)$ is dense and for which the mapping

$$
\left(d d^{c}\right)^{k}: L^{\infty}(\Omega) \cap P(\Omega) \rightarrow M_{k}
$$

is continuous. 
Another approach to defining the Monge-Ampere operator which does not involve integration by parts will be given in Section 5 .

\section{The Minimum Principle}

The uniqueness part of Theorem $D$ is an immediate consequence of Theorem A, which we prove in this section. We note that for the case of $C^{2}$ functions, another proof has been given by Kerzman, Kohn, and Nirenberg [15].

The proof of Theorem $\mathrm{A}$ is based on the following inequality.

Proposition 3.1. Let $G$ be a bounded open set in $\mathbb{C}^{n}$ with smooth boundary, and let $u, v \in C^{2}(\bar{G})$ be plurisubharmonic functions on $G$. If $u=v$ on $\partial G$ and $u \geqq v$ in $G$, then

$$
\int_{G}\left(d d^{c} u\right)^{n} \leqq \int_{G}\left(d d^{c} v\right)^{n} .
$$

Proof. One may factor

$$
\begin{aligned}
\left(d d^{c} u\right)^{n}-\left(d d^{c} v\right)^{n}= & \left(d d^{c} u-d d^{c} v\right) \wedge\left(\left(d d^{c} u\right)^{n-1}+\left(d d^{c} u\right)^{n-2} \wedge d d^{c} v\right. \\
& \left.+\cdots+\left(d d^{c} v\right)^{n-1}\right)=d d^{c}(u-v) \wedge \theta
\end{aligned}
$$

where $\theta$ is a positive, closed $(n-1, n-1)$ form. In particular, $d \rho \wedge d^{\mathrm{c}} \rho \wedge \theta \geqq 0$ for all $C^{1}$ functions $\rho$ (see e.g. [17], p. 68). Thus, by Stokes' theorem,

$$
\int_{G}\left(d d^{c} u\right)^{n}-\left(d d^{c} v\right)^{n}=\int_{\partial G} d^{c}(u-v) \wedge \theta .
$$

Let $\rho \in C^{\infty}$ be a function which defines $\partial G$. That is, $G=\{\rho<0\}, \partial G=\{\rho=0\}$, and $\nabla \rho \neq 0$ on $\partial G$. Then since $u-v$ vanishes on $\partial G$, we have $u-v=\alpha \rho$, where $\alpha \leqq 0$ in $\bar{G}$, since $u \geqq v$ and $\rho<0$ in $G$. Thus, on $\partial G$,

$$
d^{c}(u-v)=d^{c}(\alpha \rho)=\alpha d^{c} \rho \quad \text { so } \int_{G}\left(d d^{c} u\right)^{n}-\left(d d^{c} v\right)^{n}=\int_{\partial G} \alpha d^{c} \rho \wedge \theta .
$$

But, if $\sigma$ is the surface area on $\partial G$, then we have $\sigma=\frac{* d \rho}{\|d \rho\|}$, where $*$ is the Hodge star operator on $\mathbb{R}^{2 n}=C^{n}$. Thus, if $\alpha d^{c} \rho \wedge \theta=f d \sigma$, then $\alpha d \rho \wedge d^{c} \rho \wedge \theta=f d \rho \wedge \frac{* d \rho}{\|d \rho\|}$.
But since $\alpha \leqq 0$ and $d \rho \wedge d^{c} \rho \wedge \theta \geqq 0$, we must have that $f \leqq 0$. Hence,

$$
\int_{G}\left(d d^{c} u\right)^{n}-\left(d d^{c} v\right)^{n}=\int_{\partial G} f d \sigma \leqq 0
$$

since $f \leqq 0$. This completes the proof.

Our method allows us to prove the following slight extension of Theorem A.

Theorem 3.2. Let $\Omega$ be a bounded open set in $\mathbb{C}^{n}$. Let $v$ be a continuous function on $\bar{\Omega}$ which is plurisubharmonic on $\Omega$. Let $u$ be a plurisubharmonic function on $\Omega$ such that

$$
\lim _{\zeta \rightarrow z \in \partial G} \inf u(\zeta)-v(\zeta) \geqq 0 ;
$$

and

$$
\lim _{\varepsilon \rightarrow 0}\left(d d^{c} u_{\varepsilon}\right)^{n} \leqq\left(d d^{c} v\right)^{n} \text { in } \Omega,
$$


where $u_{\varepsilon}=u * \chi_{\varepsilon}$ and $\chi_{\varepsilon}$ is a usual smoothing kernel for plurisubharmonic functions ([17], p. 45). Then $u \geqq v$ in $\Omega$.

Proof. Assume the theorem is false. That is, there exists $z_{0} \in \Omega$ such that $u\left(z_{0}\right)<v\left(z_{0}\right)$. Let $\eta_{0}=\frac{1}{2}\left(v\left(z_{0}\right)-u\left(z_{0}\right)\right)$. Then for all $0<\eta<\eta_{0}$, the set

$$
G(\eta)=\{z \in \Omega: u(z)+\eta<v(z)\} \ni z_{0}
$$

is a nonempty, open ( $u-v$ is upper semicontinuous), relatively compact subset of $\Omega$, because of hypothesis (1).

Let $u_{\varepsilon}=u * \chi_{\varepsilon}, v_{\varepsilon}=v * \chi_{\varepsilon}$ be regularizations of $u, v$ as in hypothesis (2), so that $u_{\varepsilon}, v_{\varepsilon}$ are defined on

$$
\Omega_{\varepsilon}=\{z \in \Omega \mid \text { distance from } z \text { to } \partial \Omega \text { exceeds } \varepsilon\}
$$

(i.e. $\chi_{\varepsilon}$ is supported in $|z| \leqq \varepsilon$ ), and $u_{\varepsilon} \geqq u, v_{\varepsilon} \geqq v$. Since $v$ is continuous, $v_{\varepsilon} \rightarrow v$ uniformly on compact subsets of $\Omega$.

Define

$$
G(\eta, \delta)=\left\{z \in \Omega: u(z)+\eta<v(z)+\delta\left|z-z_{0}\right|^{2}\right\} .
$$

There exists a function $\delta(\eta)>0,0<\eta<\eta_{0}$, which is increasing and such that $G(\eta, \delta)$ is nonempty, open, and relatively compact in $\Omega$ for all $0<\delta \leqq \delta(\eta)$. We have, in fact $z_{0} \in G(\eta, \delta)$. Next, choose $\varepsilon(\eta, \delta)>0$ so small that $0<\varepsilon<\varepsilon(\eta, \delta)$ implies

$$
\Omega_{\varepsilon} \supset G(\eta / 2, \delta), \quad 0<\eta<\eta_{0}, 0<\delta<\delta(\eta / 2) .
$$

Then define, for such $\varepsilon, \eta, \delta$,

$$
G(\eta, \delta, \varepsilon)=\left\{z \in G(\eta / 2, \delta): u(z)+\eta<v_{\varepsilon}(z)+\delta\left|z-z_{0}\right|^{2}\right\} .
$$

If $\varepsilon$ is so small that $\left|v(z)-v_{\varepsilon}(z)\right| \leqq \eta / 4$ whenever $z \in G(\eta / 2, \delta)$ and $\varepsilon<\varepsilon(\eta, \delta)$, then

$$
G(\eta, \delta, \varepsilon) \subset G(3 \eta / 4, \delta) \subset G(\eta / 2, \delta)
$$

because $z \in G(\eta / 2, \delta) \backslash G(3 \eta / 4, \delta)$ implies

$$
\begin{aligned}
u_{\varepsilon}(z)+\eta & \geqq u(z)+\frac{3}{4} \eta+\frac{\eta}{4} \geqq v(z)+\delta\left|z-z_{0}\right|^{2}+\frac{\eta}{4} \\
& \geqq v_{\varepsilon}(z)+\delta\left|z-z_{0}\right|^{2}-\left|v(z)-v_{\varepsilon}(z)\right|+\frac{\eta}{4} \\
& \geqq v_{\varepsilon}(z)+\delta\left|z-z_{0}\right|^{2} .
\end{aligned}
$$

In particular, $G(\eta, \delta, \varepsilon)$ is a relatively compact subset of $\Omega_{\varepsilon}$, so $v_{\varepsilon}$ is $C^{\infty}$ in a neighborhood of the closure of $G(\eta, \delta, \varepsilon)$.

Finally, choose $\tau(\eta, \delta, \varepsilon)$ so small that for $\eta, \delta, \varepsilon$ as above and $0<\tau<\tau(\eta, \delta, \varepsilon)$ we have that

$$
G(\eta, \delta, \varepsilon, \tau)=\left\{z \in G\left(\frac{1}{2} \eta, \delta\right): u_{\tau}(z)+\eta<v_{\varepsilon}(z)+\delta\left|z-z_{0}\right|^{2}\right\}
$$

is a nonempty, open, relatively compact subset of $\Omega_{\varepsilon}$. Because $u_{\tau} \geqq u$, we have $G(\eta, \delta, \varepsilon, \tau) \subset G(\eta, \delta, \varepsilon)$ and because $z_{0} \in G(\eta, \delta, \varepsilon)$, we have $z_{0} \in G(\eta, \delta, \varepsilon, \tau)$ when $\tau$ is sufficiently small.

We now want to apply Proposition 3.1 with $G=G(\eta, \delta, \varepsilon, \tau)$ and the functions defining this set. The only problem is that $G$ may not have a smooth boundary. 
However, by Sard's theorem, the value $-\eta$ is a regular value of the $C^{\infty}$ function $u_{\tau}(z)-v_{\varepsilon}(z)-\delta\left|z-z_{0}\right|^{2}$ for almost all values of $\eta$. Thus, we can take a sequence of numbers $\tau_{n} \rightarrow 0$ and apply the Proposition to $G$ for almost all values of $\eta$. Consequently we have by Proposition 3.1 and (2) of Proposition 2.8,

$$
\begin{aligned}
\int\left[d d^{c} u_{\tau}\right]^{n} & =\int\left[d d^{c}\left(u_{\tau}+\eta\right)\right]^{n} \geqq \int\left[d d^{c}\left(v_{\varepsilon}+\delta\left|z-z_{0}\right|^{2}\right)\right]^{n} \\
& \geqq \int\left[d d^{c} v_{\varepsilon}\right]^{n}+\delta^{n} \int\left[d d^{c}\left|z-z_{0}\right|^{2}\right]^{n} \\
& =\int\left[d d^{c} v_{\varepsilon}\right]^{n}+\delta^{n} \cdot 4^{n} n ! \operatorname{vol} G(\eta, \delta, \varepsilon, \tau)
\end{aligned}
$$

where all integrals are over $G=G(\eta, \delta, \varepsilon, \tau)$. Letting $\tau \rightarrow 0$, the open sets $G(\eta, \delta, \varepsilon, \tau)$ increase to $G(\eta, \delta, \varepsilon)$. If $\mu=\lim \left(d d^{c} u_{\imath}\right)^{n}$, then we deduce from (3.1) that

$$
\mu(G(\eta, \delta, \varepsilon)) \geqq \int_{G(\eta, \delta, \varepsilon)}\left[d d^{c} v_{\varepsilon}\right]^{n}+n ! 4^{n} \delta^{n} \operatorname{vol} G(\eta, \delta, \varepsilon)
$$

for almost all $\eta<\eta_{0}, 0<\delta<\delta(\eta)$, and $0<\varepsilon<\varepsilon(\eta, \delta)$. Now let $\varepsilon \rightarrow 0$. The measures $\left[d d^{c} v_{\varepsilon}\right]^{n}$ converge weakly to $\left[d d^{c} v\right]^{n}$, by Proposition 2.3. Also

$$
\begin{aligned}
G(\eta, \delta, \varepsilon) & \supset\left\{z \in G\left(\frac{1}{2} \eta, \delta\right): u(z)+\eta<v(z)+\delta\left|z-z_{0}\right|^{2}\right\} \\
& =G\left(\frac{1}{2} \eta, \delta\right) \cap G(\eta, \delta)=G(\eta, \delta) .
\end{aligned}
$$

Further,

$$
\bigcap_{\varepsilon>0} G(\eta, \delta, \varepsilon) \subset K(\eta, \delta)=\left\{z \in \Omega: u(z)+\eta \leqq v(z)+\delta\left|z-z_{0}\right|^{2}\right\} .
$$

Thus, we have for almost all $\eta$,

$$
\mu(K(\eta, \delta)) \geqq \int_{G(\eta, \delta)}\left(d d^{c} v\right)^{n}+n ! 4^{n} \delta^{n} \cdot \operatorname{vol} G(\eta, \delta) .
$$

Since $\mu \leqq\left(d d^{c} v\right)^{n}$, this implies, with $v=\left(d d^{\mathfrak{c}} v\right)^{n}$,

$$
v(K(\eta, \delta)) \geqq v(G(\eta, \delta))+n ! 4^{n} \delta^{n} \cdot \operatorname{vol} G(\eta, \delta) .
$$

However, $\eta \rightarrow v(G(\eta, \delta))$ is an increasing function of $\eta$, and $K(\eta, \delta) \subset G\left(\eta^{\prime}, \delta\right)$ for $\eta^{\prime}>\eta$. At points of continuity of this function we then have

$$
v(G(\eta, \delta))=v(G(\eta, \delta))+n ! 4^{n} \delta^{n} \operatorname{vol} G(\eta, \delta)
$$

which contradicts the fact that $G(\eta, \delta)$ is a nonempty, open, relatively compact subset of $\Omega$. This completes the proof.

Corollary 3.3. Let $\Omega$ be a bounded open set in $\mathbb{C}^{n}$ and $\phi$ a continuous, real-valued function on $\partial \Omega$. The solution, if one exists, of the Dirichlet problem

$$
\begin{array}{ll}
u \text { plurisubharmonic in } \Omega \\
u \text { continuous } & \text { in } \bar{\Omega} \\
u=\phi & \text { in } \partial \Omega \\
\left(d d^{c} u\right)^{n}=\mu & \text { in } \Omega
\end{array}
$$

where $\mu$ is a given positive Borel measure, is unique.

Proof. If $u, v$ are two solutions of (3.3), we have $u \geqq v$ and $v \geqq u$ in $\Omega$ by Theorem A. 
Finally, we note as a consequence of Theorem 3.2 the following very weak regularity theorem for the equation $\left(d d^{c} u\right)^{n}=0$.

Theorem 3.4. Let $\Omega$ be a strictly pseudoconvex bounded open set in $\mathbb{C}^{n}$. Suppose that $u$ is a plurisubharmonic function in $\Omega$ such that

(1) $\lim _{\varepsilon \rightarrow 0}\left(d d^{c} u_{\varepsilon}\right)^{n}=0,\left(u_{\varepsilon}=u * \chi_{\varepsilon}\right.$ as in (2) of Theorem 3.2) and

(2) there is a continuous function $\phi: \partial \Omega \rightarrow R$ with

$$
\lim _{\zeta \rightarrow z \in \partial \Omega} u(\zeta)=\phi(z)
$$

Then $u$ is continuous in $\bar{\Omega}$.

Proof. From Theorem 3.2 it follows $u$ is the upper envelope of all plurisubharmonic functions on $\Omega$ which are continuous on $\bar{\Omega}$ and are $\leqq \phi$ on $\partial \Omega$. By a theorem of J.B. Walsh ([23], p. 145), $u$ is continuous.

\section{Examples of Nonuniqueness in the Dirichlet Problem}

In this section we give examples of nonuniqueness in the Dirichlet problem (1). This is associated with the failure of the minimum principle, Theorem A, and we first give simple examples where this principle fails. In all cases, it is of course necessary to go outside the class of continuous plurisubharmonic functions. We will restrict ourselves to the case $n=2$, and the examples given here fall under the case $\nabla u$ locally $L^{2}$, which was discussed in Section 2 (or a simple modification can be made to bring them into this case). It will be clear how corresponding examples for $n>2$ may be found.

We will consider functions of the form

$$
u(z, w)=\frac{1}{2} \log \left(|f(z, w)|^{2}+|g(z, w)|^{2}\right)
$$

where $f, g$ are analytic. If $|f|+|g|>0$, then we compute

$$
\begin{aligned}
& \bar{\partial} u=\frac{f \overline{\partial f}+g \overline{\partial g}}{2\left(|f|^{2}+|g|^{2}\right)}, \\
& \partial \bar{\partial} u=\frac{(f \partial g-g \partial f) \wedge(\overline{f \partial g-g \partial f})}{2\left(|f|^{2}+|g|^{2}\right)^{2}}, \bar{\partial} \wedge \overline{\partial g} \\
& \bar{\partial} u \wedge \partial \bar{\partial} u=\frac{-(f \partial g-g \partial f) \wedge \overline{\partial f}}{4\left(|f|^{2}+|g|^{2}\right)^{2}}, \\
& \partial \bar{\partial} u \wedge \partial \bar{\partial} u=0 .
\end{aligned}
$$

However, if $f$ and $g$ can both vanish, it is not true that $\left(d d^{c} u\right)^{2}=-4 \partial \bar{\partial} u \wedge \partial \bar{\partial} u$ vanishes. Let $F=(f, g)$ be thought of as a map of $\mathbb{C}^{2} \rightarrow \mathbb{C}^{2}$. If $F$ has an isolated zero at $(0,0) \in \mathbb{C}^{2}$, then near $(0,0)$,

$$
\left(d d^{c} u\right)^{2}=4 \pi^{2} p \delta
$$


where $p$ is the degree of $F$ at $(0,0)$ and $\delta$ is the unit point mass measure at $(0,0)$. This can be seen in a couple of ways. One way is to note that the restriction of the form

$$
\omega=d^{c}\left[\frac{1}{2} \log \left(|z|^{2}+|w|^{2}\right)\right] \wedge d d^{c}\left[\frac{1}{2} \log \left(|z|^{2}+|w|^{2}\right)\right]
$$

to a sphere $S_{\varepsilon}=\left\{|z|^{2}+|w|^{2}=\varepsilon^{2}\right\}$ is equal to $2 \varepsilon^{-3} d \sigma_{\varepsilon}$, where $d \sigma_{\varepsilon}$ is the volume form on the sphere $S_{\varepsilon}$. The form $d^{c} u \wedge d d^{c} u$ is the pullback under $F=(f, g)$ of the form $\omega$. Further, $\int F^{*}\left(\varepsilon^{-3} d \sigma_{\varepsilon}\right)=2 \pi^{2} p$, where $p=$ degree of $F$ at $(0,0)$. Thus,

$$
\int_{|z|^{2}+|w|^{2}=\varepsilon^{2}} d^{c} u \wedge d d^{c} u=2 \int F^{*}\left(\varepsilon^{-3} d \sigma_{\varepsilon}\right)=4 \pi^{2} p
$$

which, together with (4.4), gives (4.5).

Another way to see (4.5) is to notice that $d^{c} u \wedge d d^{c} u$ is essentially the CauchyFantappiè form associated to the map $F$, and the value of $\int d^{c} u \wedge d d^{c} u$ follows from well-known integral formulas (c.f. [16]).

Example I. For an example where Theorem A fails, set

$$
u_{1}(z, w)=\frac{1}{2} \log \left(|z|^{2}+|w|^{2}\right)
$$

and

$$
u_{2}(z, w)=\frac{1}{2 \sqrt{2}} \log \left(|z|^{2}+|w|^{4}\right) .
$$

By (4.5), we have

$$
\left[d d^{c} u_{1}\right]^{2}=\left[d d^{c} u_{2}\right]=4 \pi^{2} \delta .
$$

However, there are points inside the unit ball $|z|^{2}+|w|^{2}<1$ where $u_{1}>u_{2}$ (e.g. where $w=0$ ) and points where $u_{2}>u_{1}$ (e.g. where $z=0$ ), even though $u_{1} \geqq u_{2}$ for all $(z, w)$ with $|z|^{2}+|w|^{2}=1$.

It is also in general false that

$$
\left(d d^{c}[\max (u, v)]\right)^{2} \geqq \min \left(\left(d d^{c} u\right)^{2},\left(d d^{c} v\right)^{2}\right)
$$

in contrast to (1) of Proposition 2.8 for continuous functions, as the following example shows.

Example II. Set $u_{1}(z, w)=\frac{1}{2} \log \left(|z|^{2}+|w|^{4}\right)$ and $u_{2}(z, w)=u_{1}(w, z)=\frac{1}{2} \log \left(|z|^{4}+|w|^{2}\right)$. Let

$$
U(z, w)=\max \left(u_{1}, u_{2}\right) .
$$

If $\Omega_{1}=\left\{(z, w):|z|^{2}+|w|^{2}<1,0<|w|<|z|\right\}$ and $\Omega_{2}=\left\{(z, w):|z|^{2}+|w|^{2}<1,0<|z|<|w|\right\}$, then we easily verify that $U=u_{i}$ on $\Omega_{i}$.

We claim that $\left(d d^{c} U\right)^{2}$ is equal to $4 \pi^{2} \delta$ plus the restriction to $\partial \Omega_{1} \backslash\{(0,0)\}$ of the 3-form $\left(d^{c} u_{1}-d^{c} u_{2}\right) \wedge\left(d d^{c} u_{1}+d d^{c} u_{2}\right)$. That is, if $\phi$ is a $C^{\infty}$ function with compact support in $|z|^{2}+|w|^{2}<1$, then

$$
\int \phi\left(d d^{c} U\right)^{2}=4 \pi^{2} \phi(0)+\int_{|w|=|z|>0} \phi d^{c}(u-v) \wedge d d^{c}(u+v) .
$$

In particular, (4.6) fails, since by (4.5), $\min \left(d d^{c} u_{i}\right)^{2}=8 \pi^{2} \delta$. 
Before proving (4.7), let us note some facts about the various weak derivatives of $U$. The first derivatives of $U$ are functions, and we have

$$
d U=d u_{i}, \quad d^{c} U=d^{c} u_{i} \quad \text { on } \Omega_{i}, \quad i=1,2
$$

as is easily verified using formula (4.1). Since $d^{c} U$ is smooth except at zero and except for the jump across the hypersurface $0<|z|=|w|$, it is then standard to compute that

$$
d d^{c} U=v+\eta
$$

where $v, \eta$ are the currents, $v=d d^{c} u_{i}$ on $\Omega_{i}, i=1,2$ and $\eta$ is the restriction of $d^{c} u_{2}-d^{c} u_{1}$ to the part of the boundary of $\Omega_{1}$ where $0<|z|=|w|$. That is, for all smooth $(1,1)$ forms $\phi$ with compact support in $|z|^{2}+|w|^{2}<1$

$$
\int \phi \wedge d d^{c} U=\int_{\partial \Omega_{1} \backslash\{(0,0)\}} \phi \wedge\left(d^{c} u_{2}-d^{c} u_{1}\right)+\sum_{i=1}^{2} \int_{\Omega_{1}} \phi \wedge d d^{c} u_{i} .
$$

To compute $\left(d d^{c} U\right)^{2}$, we can use either (1) or (2) of Proposition 2.1.

$$
\begin{aligned}
\int \phi\left(d d^{c} U\right)^{2} & =-\int d d^{c} \phi \wedge d U \wedge d^{c} U \\
& =\int U d d^{c} \phi \wedge d d^{c} U .
\end{aligned}
$$

To be explicit, let us use the first of these formulas. Let $\Omega_{i}(\varepsilon)=\Omega_{i} \cap\left\{|z|^{2}+|w|^{2}>\varepsilon^{2}\right\}$, $M_{i}(\varepsilon)=\partial \Omega_{i}(\varepsilon) \cap\left\{|z|^{2}+|w|^{2}>\varepsilon^{2}\right\}$ and $S_{i}(\varepsilon)=\partial \Omega_{i}(\varepsilon) \cap\left\{|z|^{2}+|w|^{2}=\varepsilon^{2}\right\}$. Then by (4.8),

$$
\begin{aligned}
\int \phi\left(d d^{c} U\right)^{2} & =-\sum_{i=1}^{2} \int_{\Omega_{i}} d d^{c} \phi \wedge d u_{i} \wedge d^{c} u_{i} \\
& =\lim _{\varepsilon \rightarrow 0} \sum_{i=1}^{2} \int_{\Omega_{i}(\varepsilon)} d d^{c} \phi \wedge d u_{i} \wedge d^{c} u_{i} .
\end{aligned}
$$

We want to move the differentiations off of $\phi$. Thus,

$$
\begin{aligned}
\int_{\Omega_{i}(\varepsilon)} d d^{c} \phi \wedge d u_{i} \wedge d^{c} u_{i}= & -\int_{\Omega_{i}(\varepsilon)} d^{c} \phi \wedge d u_{i} \wedge d d^{c} u_{i} \\
& +\int_{\partial \Omega_{i}(\varepsilon)} d^{c} \phi \wedge d u_{i} \wedge d^{c} u_{i} \\
= & \int_{\Omega_{i}(\varepsilon)} d \phi \wedge d^{c} u_{i} \wedge d d^{c} u_{i}+\int_{\partial \Omega_{i}(\varepsilon)} d^{c} \phi \wedge d u_{i} \wedge d^{c} u_{i} \\
& =\int_{\partial \Omega_{i}(\varepsilon)} \phi d^{c} u_{i} \wedge d d^{c} u_{i}+d^{c} \phi \wedge d u_{i} \wedge d^{c} u_{i}
\end{aligned}
$$

since $d d^{c} u_{i} \wedge d d^{c} u_{i} \equiv 0$ on $\Omega_{i}(\varepsilon)$. Now use formulas (4.1) and (4.3) to compute $d^{c} u_{i} \wedge d d^{c} u_{i}, d u_{i} \wedge d^{c} u_{i}$. We find that on $S_{i}(\varepsilon)$,

$$
\left|d^{c} u_{i} \wedge d d^{c} u_{i}\right| \leqq \text { const. } / \varepsilon, \quad\left|d u_{i} \wedge d^{c} u_{i}\right| \leqq \text { const. } / \varepsilon^{2}
$$

so that

$$
\left|\int_{S_{i}(\varepsilon)} d^{c} u_{i} \wedge d d^{c} u_{i}+\int_{S_{i}(\varepsilon)} d^{c} \phi \wedge d u_{i} \wedge d^{c} u_{i}\right| \leqq 0\left(\varepsilon^{2}\right)+0(\varepsilon) \rightarrow 0 .
$$


Therefore

$$
\begin{aligned}
\int \phi\left(d d^{c} U\right)^{2} & =\lim _{\varepsilon \rightarrow 0} \sum_{i=1}^{2} \int_{M_{i}(\varepsilon)} \phi d^{c} u_{i} \wedge d d^{c} u_{i}+d^{c} \phi \wedge d u_{i} \wedge d^{c} u_{i} \\
& =\lim _{\varepsilon \rightarrow 0}\left\{\int_{M_{1}(\varepsilon)}\left[d^{c} u_{1} \wedge d d^{c} u_{1}-d^{c} u_{2} \wedge d d^{c} u_{2}\right]+\int_{M_{1}(\varepsilon)} d^{c} \phi \wedge d u_{1} \wedge d^{c}\left(u_{1}-u_{2}\right)\right\}
\end{aligned}
$$

where the last equality results from $M_{1}(\varepsilon), M_{2}(\varepsilon)$ having opposite orientations, and $d u_{1}=d u_{2}$ on $M_{1}(\varepsilon)$, because $u_{1}=u_{2}=0$ defines $\partial \Omega_{1}$.

Now, on the manifold $\left\{u_{1}=u_{2}\right\} \backslash(0,0) \supset M_{1}(\varepsilon)$, we have $d^{c} \phi \wedge d u_{1} \wedge d^{c}\left(u_{1}-u_{2}\right)$ $=-d \phi \wedge d^{c} u_{1} \wedge d^{c}\left(u_{1}-u_{2}\right)$ because the $(1,1)$ parts of $d^{c} \phi \wedge d u_{1}$ and $-d \phi \wedge d^{c} u_{1}$ are the same so

$$
d\left(u_{1}-u_{2}\right) \wedge d^{c} \phi \wedge d u_{1} \wedge d^{c}\left(u_{1}-u_{2}\right)=-d\left(u_{1}-u_{2}\right) \wedge d \phi \wedge d^{c} u_{1} \wedge d^{c}\left(u_{1}-u_{2}\right) .
$$

Thus,

$$
\begin{aligned}
\int_{M_{1}(\varepsilon)} d^{c} \phi \wedge d u_{1} \wedge d^{c}\left(u_{1}-u_{2}\right)= & -\int_{M_{1}(\varepsilon)} d \phi \wedge d^{c} u_{1} \wedge d^{c}\left(u_{1}-u_{2}\right) \\
= & -\int_{M_{1}(\varepsilon)} d \phi \wedge d^{c} u_{1} \wedge d^{c} u_{2} \\
= & -\int_{M_{1}(\varepsilon)} d\left[\phi d^{c} u_{1} \wedge d^{c} u_{2}\right] \\
& -\int_{M_{1}(\varepsilon)} \phi\left(d^{c} u_{2} \wedge d d^{c} u_{1}-d^{c} u_{1} \wedge d d^{c} u_{2}\right)
\end{aligned}
$$

Together with (4.13), this implies

$$
\begin{aligned}
\int \phi\left(d d^{c} U\right)^{2}= & \lim _{\varepsilon \rightarrow 0} \int_{M_{1}(\varepsilon)} \phi d^{c}\left(u_{1}-u_{2}\right) \wedge d d^{c}\left(u_{1}+u_{2}\right) \\
& -\int_{M_{1}(\varepsilon)} d\left[\phi d^{c} u_{1} \wedge d^{c} u_{2}\right] .
\end{aligned}
$$

However,

$$
-\int_{M_{1}(\varepsilon)} d\left[\phi d^{c} u_{1} \wedge d^{c} u_{2}\right]=-\int_{\partial M_{1}(\varepsilon)} \phi d^{c} u_{1} \wedge d^{c} u_{2}
$$

So

$$
\lim _{\varepsilon \rightarrow 0}-\int_{\partial M_{1}(\varepsilon)} \phi d^{c} u_{1} \wedge d^{c} u_{2}=-\phi(0) \lim _{\varepsilon \rightarrow 0} \int_{\partial M_{1}(\varepsilon)} d^{c} u_{1} \wedge d^{c} u_{2} .
$$

Finally, direct computation shows that $\int_{\partial M_{1}(\varepsilon)} d^{c} u_{1} \wedge d^{c} u_{2}=-4 \pi^{2}+0\left(\varepsilon^{2}\right)$, so (4.7) follows from (4.14).

To construct an example of nonuniqueness in the Dirichlet problem, we will make use of a relationship between the complex and real Monge-Ampere operators. Namely, suppose $U(z)=U\left(z_{1}, \ldots, z_{n}\right)=U\left(\left|z_{1}\right|, \ldots,\left|z_{n}\right|\right)$. If we set $\left|z_{i}\right|=e^{x_{i}}$ and

$$
f\left(x_{1}, \ldots, x_{n}\right)=U\left(e^{x_{1}}, \ldots, e^{x_{n}}\right)
$$

then it is easily checked that

$$
4 z_{i} \bar{z}_{j} \frac{\partial^{2} U}{\partial z_{i} \partial \bar{z}_{j}}=\frac{\partial^{2} f}{\partial x_{i} \partial x_{j}}
$$


so

$$
M f \stackrel{\text { def }}{=} \operatorname{det}\left[\frac{\partial^{2} f}{\partial x_{i} \partial x_{j}}\right]=4^{n}\left|z_{1}\right|^{2} \ldots\left|z_{n}\right|^{2} \operatorname{det}\left[\frac{\partial^{2} U}{\partial z_{i} \partial \bar{z}_{j}}\right] .
$$

With examples of the form $u\left(\left|z_{1}\right|, \ldots,\left|z_{n}\right|\right)$ in mind, we will now construct a family of functions in two variables such that

$$
\begin{array}{rlrl}
\left(d d^{c} u\right)^{2} & =c \delta & & \text { on }|z|^{2}+|w|^{2}<1, \\
u & =0 & \text { on }|z|^{2}+|w|^{2}=1
\end{array}
$$

where $\delta$ is the point mass measure at the origin. These will be functions $u(|z|,|w|)$ so that the associated convex functions

$$
f(x, y)=u\left(e^{x}, e^{y}\right)
$$

have $M f=0$ on

$$
\Omega^{*}=\left\{(x, y): z=e^{x}, w=e^{y},|z|^{2}+|w|^{2}<1\right\}
$$

but, $M f$ has some "mass at infinity".

Fix positive numbers $p, q$. We will take $f$ in the form

$$
f(x, y)=\left(p^{2}+q^{2}\right)^{-1 / 2}(q x+p y)-\phi(q y-p x)
$$

where $\phi$ is a suitable smooth function. Now, we want $f=0$ on $\partial \Omega^{*}$. Each line of slope $p / q$ intersects $\partial \Omega^{*}$ in exactly one point. Thus, there is a map $\Phi: \mathbb{R}^{2} \rightarrow \partial \Omega^{*}$, say $\Phi(x, y)=\left(x^{\prime}, y^{\prime}\right)$ given by projecting $(x, y)$ along the line of slope $p / q$ passing through $(x, y)$ to the intersection of the line with $\partial \Omega^{*}$. The mapping $\Phi$ is smooth. The function $f$ is linear with slope +1 on each line of slope $p / q$, and $\phi(q y-p x)$ is constant on such lines. Thus, if $f$ is to vanish on $\partial \Omega^{*}$, we have

$$
\phi(q y-p x)=-\frac{q x^{\prime}+p y^{\prime}}{\left(p^{2}+q^{2}\right)^{1 / 2}}
$$

or

$$
f(x, y)=\frac{q\left(x-x^{\prime}\right)+p\left(y-y^{\prime}\right)}{\left(p^{2}+q^{2}\right)^{1 / 2}} .
$$

We can also compute the derivatives of $f$. To do this, it suffices to compute the Jacobian matrix of $\Phi$. This is a direct computation which we omit. The results are as follows. Set $a=e^{2 x^{\prime}}, b=e^{2 y^{\prime}}, \Delta=(p b+q a)^{-1}$. Then with

$$
\begin{aligned}
& \alpha_{1}=\frac{\partial x^{\prime}}{\partial x}, \quad \alpha_{2}=\frac{\partial x^{\prime}}{\partial y}, \quad \alpha_{3}=\frac{\partial y^{\prime}}{\partial x}, \quad \alpha_{4}=\frac{\partial y^{\prime}}{\partial y}, \\
& \alpha_{1}=p b \Delta, \quad \alpha_{2}=-b q \Delta, \quad \alpha_{3}=-p a \Delta, \quad \alpha_{4}=a q \Delta .
\end{aligned}
$$

The second derivatives can be computed in the same way. A typical result is

$$
\frac{\partial \alpha_{1}}{\partial x}=-2 a b p^{2} q \Delta^{3}
$$

The only important point for us is that each of these second derivatives has the form $a b H$, where $H$ is a polynomial in $a, b, p, q$, and $\Delta$. 
Let us note some consequences of formulas (4.20) and (4.21). First, since $a+b=1$, we have $0<\Delta \leqq \min \left(p^{-1}, q^{-1}\right)$, so

$$
\begin{array}{ll}
\left|\alpha_{i}\right| \leqq \text { const. } b, & i=1,2, \\
\left|\alpha_{i}\right| \leqq \text { const. } a, & i=3,4 .
\end{array}
$$

Also, if $D^{2} f$ is any second partial derivative of $f$, we have

$$
\left|D^{2} f\right| \leqq \text { const. } a b
$$

For later reference, we also want to compute some special derivatives of $f$. Let $\bar{n}=\left(p^{2}+q^{2}\right)^{-1 / 2}(q, p)$ be a unit vector having the direction of lines with slope $p / q$, and $\bar{t}=\left(p^{2}+q^{2}\right)^{-1 / 2}(-p, q)$ the vector orthogonal to $\bar{n}$. Introduce new coordinates $(\xi, \eta)$ on $\mathbb{R}^{2}$ so that $(x, y)=\xi \bar{n}+\eta \bar{t}$. That is,

$$
x=\left(p^{2}+q^{2}\right)^{-1 / 2}(q \xi-p \eta), \quad y=\left(p^{2}+q^{2}\right)^{-1 / 2}(p \xi+q \eta) .
$$

In this coordinate system, we find, using the chain rule and our previous calculations

$$
\frac{\partial f}{\partial \xi}=1, \quad \frac{\partial f}{\partial \eta}=\frac{q b-p a}{p b+q a}
$$

Note that if $x \rightarrow-\infty$ while $y$ remains bounded, then $x^{\prime} \rightarrow-\infty, y^{\prime} \rightarrow 0$, so $a \rightarrow 0$, $b \rightarrow 1$. Thus.

$$
\lim _{\substack{x \rightarrow-\infty \\ y \text { bounded }}} \frac{\partial f}{\partial \eta}=\frac{q}{p}
$$

Similarly

$$
\lim _{\substack{y \rightarrow-\infty \\ x \text { bounded }}} \frac{\partial f}{\partial \eta}=-\frac{p}{q} .
$$

Proposition 4.1. Let $\omega=\frac{\partial f}{\partial x} d\left(\frac{\partial f}{\partial y}\right)-\frac{\partial f}{\partial y} d\left(\frac{\partial f}{\partial x}\right)$. Then

$$
\int \underset{\partial \Omega^{*}}{\omega}=\frac{p^{2}+q^{2}}{p q} .
$$

Proof. The estimates given for first and second derivatives of $f$ show that the integral converges. To evaluate ot, transform to $(\xi, \eta)$ coordinates. Then

$$
\omega=\frac{\partial f}{\partial \xi} d\left(\frac{\partial f}{\partial \eta}\right)-\frac{\partial f}{\partial \eta} d\left(\frac{\partial f}{\partial \xi}\right)=d\left(\frac{\partial f}{\partial \eta}\right)
$$

by (4.25). Thus,

$$
\int_{\partial \Omega^{*}} \omega=\lim _{x^{\prime} \rightarrow-\infty, y^{\prime} \rightarrow 0} \frac{\partial f}{\partial \eta}-\lim _{x^{\prime} \rightarrow 0, y^{\prime} \rightarrow-\infty} \frac{\partial f}{\partial \eta}
$$

which, by (4.26) and (4.27) is equal to $\frac{q}{p}+\frac{p}{q}=\frac{p^{2}+q^{2}}{p q}$, as asserted. 
Finally, we return to the function $u$ given by (4.18). Let $B=\left\{(z, w) \in \mathbb{C}^{2}:|z|^{2}+|w|^{2}<1\right\}$.

Proposition 4.2. The function $u$ is infinitely differentiable on $\bar{B} \backslash\{z w=0\}$. Further,

(1) $u$ has a continuous extension to a $C^{2}$ function on $B \backslash\{(0,0)\}$;

(2) if $u(0,0)=-\infty$, then $u$ is plurisubharmonic on $B$;

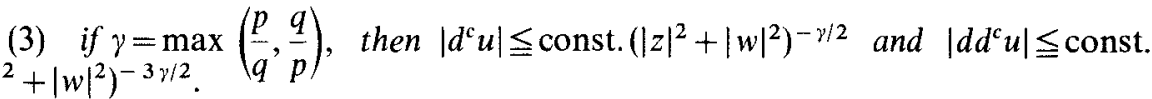

Proof. It is clear that $u$ is smooth on $\bar{B} \backslash\{z w=0\}$ since $f$ is smooth. From the formulas (4.20) and (4.21) together with the formula (4.16) for the derivatives of $u$ in terms of derivatives of $f$, it is easily checked that the derivatives of $u$ extend continuously to $\bar{B} \backslash\{(0,0)\}$. In fact, $u$ is infinitely differentiable on $\bar{B} \backslash\{(0,0)\}$, although we don't need this. To see that $u$ is plurisubharmonic on $B$, all we have to verify is that $u$ is uppersemicontinuous and locally satisfies the subaveraging property on each complex line. We omit these routine verifications.

To obtain the estimates of (3) we again use (4.20) and (4.21). First note that when $|z|>|w|$, we have $a|z|^{-2} \leqq$ const. $\left(|z|^{2}+|w|^{2}\right)^{-1}$. And, when $|z|<|w|$, we have $a|z|^{-2}=e^{2 x} e^{-2 x^{\prime}}=e^{2\left(x-x^{\prime}\right)}=e^{2 q\left(y-y^{\prime}\right) / p} \leqq|w|^{-2 q / p}$, where we have written $|z|=e^{x}$, $|w|=e^{y}$. Thus, $a /|z|^{2} \leqq$ const. $\left(|z|^{2}+|w|^{2}\right)^{-\gamma}$. Similarly $a /|z| \leqq$ const. $\left(|z|^{2}+|w|^{2}\right)^{-\gamma / 2}$. Then for a typical derivative of $u$ we have $\left|\frac{\partial u}{\partial z}\right| \leqq$ const. $|z|^{-1}\left|\frac{\partial f}{\partial x}\right| \leqq$ const. $|z|^{-1} a \leqq$ const. $\left(|z|^{2}+|w|^{2}\right)^{-\gamma / 2}$. All other derivatives are estimated in the same way.

Proposition 4.3. Suppose $p, q$ are such that $\gamma=\max \left(\frac{p}{q}, \frac{q}{p}\right)$ satisfies $\gamma<\frac{4}{3}$. Then

(1) $d^{c} u \wedge d d^{c} u$ is integrable on $B$; and

(2) $d\left[d^{c} u \wedge d d^{c} u\right]=4 \pi^{2} \frac{p^{2}+q^{2}}{p q} \delta$, where $\delta$ is the point mass measure of $(0,0)$.

Proof. From (3) of Proposition 4.2 we have that $\left|d^{c} u \wedge d d^{c} u\right| \leqq\left(|z|^{2}+|w|^{2}\right)^{-3 \gamma / 2}$, so the coefficients are integrable so long as $3 \gamma<4$. We already know that $d d^{c} u \wedge$ $d d^{c} u=0$ away from $(0,0)$, so $d d^{c} u \wedge d d^{c} u=\lambda \delta$ for some nonnegative number $\lambda$. We can compute $\lambda$ easily using Proposition 4.1. We have

$$
\lambda=\int_{|z|^{2}+|w|^{2}=1} d^{c} u \wedge d d^{c} u .
$$

A computation shows that on the unit sphere, with $x=\log r, y=\log \rho, r=|z|$, $\rho=|w|$, we have

$$
d^{c} u \wedge d d^{c} u=\left[\left(\frac{\partial f}{\partial x} \frac{\partial^{2} f}{\partial y^{2}}-\frac{\partial f}{\partial y} \frac{\partial^{2} f}{\partial x \partial y}\right) d y-\left(\frac{\partial f}{\partial y} \frac{\partial^{2} f}{\partial x^{2}}-\frac{\partial f}{\partial x} \frac{\partial^{2} f}{\partial x \partial y}\right) d x\right] \wedge d \theta \wedge d \phi .
$$

Thus, $\int_{|z|^{2}+|w|^{2}=1} d^{c} u \wedge d d^{c} u=4 \pi^{2} \int_{\partial \Omega^{*}} \omega$ where $\omega$ is the form of Proposition 4.1. Thus we find $\lambda=4 \pi^{2}\left(\frac{p^{2}+q^{2}}{p q}\right)$ as asserted. 
Remark. Note that when $p=q=1$, we have $\frac{1}{2} \log \left(|z|^{2}+|w|^{2}\right)=\frac{\sqrt{2}}{2} u$. This gives another method for computing (4.5).

Example III. Finally, to get the examples of nonuniqueness in the Dirichlet problem, consider functions of the form $\lambda u=\lambda u_{p, q}$, where $u=u_{p, q}$ is as in Proposition 4.3. We have that each such function is continuous on $\bar{B} \backslash\{0,0\}$ and vanishes for $|z|^{2}+|w|^{2}=1$. Also,

$$
\left[d d^{c}\left(\lambda u_{p, q}\right)\right]^{2}=\lambda^{2} \frac{p^{2}+q^{2}}{p q} \delta
$$

so different values of $\lambda, p, q$ can give rise to the same right-hand side.

\section{The Operator $\Phi(u)$}

We have seen that the operator $\left(d d^{c} u\right)^{n}$ has some unpleasant properties when the singularities of $u$ are too strong, for instance the failure of (4.6) and Example II of Section 4. In this section, we introduce a closely associated operator, $\Phi(u)$, which is essentially $\left[\left(d d^{c} u\right)^{n}\right]^{1 / n}$. As we will see, it has some advantages over $\left(d d^{c} u\right)^{n}$. In particular, it is defined for all plurisubharmonic functions. On the other hand, in some cases $\Phi(u)$ will fail to recognize singularities we wish to take into account (c.f. the example after Theorem 5.8).

To define $\Phi(u)$, we will use a general measure theoretic construction given by Goffman and Serrin in [12]. Let

$$
C=\left\{\xi: \xi=\left(\xi_{i j}\right) \text { is a nonnegative, } n \times n \text { Hermitian matrix }\right\}
$$

so that $C$ is a closed, convex cone. We will think of $C$ as imbedded in $\mathbb{C}^{n^{2}}=R^{4 n^{2}}$, with the usual Euclidean norm on $R^{4 n^{2}}$. Consider the function

$$
\Psi(\xi)=(\operatorname{det} \xi)^{1 / n}, \quad \xi \in C .
$$

Proposition 5.1. The function $\Psi$ is a continuous, nonnegative, concave function which is positively homogeneous of degree 1 on the cone $C$.

Proof. All assertions are clear, except that $\Psi$ is concave. To see this, let $\xi, \eta \in C$, and $0<t<1$. We have to prove

$$
\Psi(t \xi+(1-t) \eta) \geqq t \Psi(\xi)+(1-t) \Psi(\eta) .
$$

It is no loss of generality to assume that $\xi$ is nonsingular. Otherwise, replace $\xi$ by $\xi+\varepsilon I$, where $I$ is the identity matrix, and then let $\varepsilon \rightarrow 0$. Then since $\xi$ is nonsingular, we can write

$$
t \xi+(1-t) \eta=\xi^{1 / 2}\left[t I+(1-t) \xi^{-1 / 2} \eta \xi^{-1 / 2}\right] \xi^{1 / 2} .
$$

Because $\Psi(\xi \eta)=\Psi(\xi) \cdot \Psi(\eta)$, it therefore suffices to prove (5.3) when $\xi=I$. Then, if $\mu_{1}, \ldots, \mu_{n}$ are the eigenvalues of $\eta$ the inequality (5.3) is equivalent to

$$
\prod_{j=1}^{n}\left[t+(1-t) \mu_{j}\right]^{1 / n} \geqq t+(1-t)\left[\prod_{j=1}^{n} \mu_{j}\right]^{1 / n} .
$$


To prove (5.4), divide both sides by $t$, set $x=(1-t) / t$, and consider the function $f(x)=\prod_{j=1}^{n}\left(1+x \mu_{j}\right)^{1 / n}$. It suffices to prove that $f^{\prime \prime}(x) \leqq 0$ for $x>0$. A short calculation yields

$$
f^{\prime \prime}(x)=\frac{f(x)}{n^{2}}\left[\left(\sum_{j=1}^{n} \frac{\mu_{j}}{1+x \mu_{j}}\right)^{2}-n \sum_{j=1}^{n} \frac{\mu_{j}^{2}}{\left(1+x \mu_{j}\right)^{2}}\right],
$$

and the bracketed term is $\leqq 0$ by the Cauchy-Schwarz inequality.

Now, let $\mu$ be a vector valued Borel measure on $\Omega \subset \mathbb{C}^{n}$ with values in the cone $C$. That is $\mu=\left(\mu_{i j}\right)$ where the $\mu_{i j}$ are Borel measures on $\Omega$ and for each Borel set $E \subset \Omega,\left(\mu_{i j}(E)\right) \in C$. We want to define $\Psi(\mu)$, as a nonnegative Borel measure on $\Omega \subset \mathbb{C}^{n}$. This can be done in the following way. Choose a nonnegative Borel measure $\lambda$ on $\Omega$ so that $\mu$ is absolutely continuous with respect to $\lambda$. For example, $\lambda=\sum\left|\mu_{i j}\right|$, where $\left|\mu_{i j}\right|$ is the total variation measure of $\mu_{i j}$. Then by the RadonNikodym Theorem, $d \mu=h d \lambda$, where $h$ is a Borel measurable function on $\Omega$ with values in the cone $C$.

Definition 5.2. $\Psi(\mu)=\Psi(h) \lambda$.

It is routine to verify that this definition is independent of the particular Borel measure $\lambda$ used in the definition, since $\Psi$ is homogeneous of degree 1 . It is also routine to verify that $\Psi(\mu)$ is a nonnegative Borel measure, because $\Psi\left(\left[a_{i j}\right]\right) \leqq$ const. $\sum\left|a_{i j}\right|$, and $\Psi \geqq 0$.

There are several properties of the operator $\Psi$ on Borel measures which are immediate consequences of the definition.

Proposition 5.3. If $\mu, v$ are Borel measures on $\Omega$ with values in $C$, then

(1) $\Psi(\alpha \mu)=\alpha \Psi(\mu)$ if $\alpha \geqq 0$.

(2) If $\mu, v$ are mutually singular, then $\Psi(\mu+v)=\Psi(\mu)+\Psi(v)$.

(3) $\Psi(\mu)$ is absolutely continuous with respect to $\mu$.

(4) $\Psi(t \mu+(1-t) v) \geqq t \Psi(\mu)+(1-t) \Psi(v), \quad 0<t<1$.

We need also two additional facts about the operators $\Phi, \Psi$. The first is the continuous analogue of (4) of Proposition 5.3.

Proposition 5.4. If $\chi \geqq 0$ is a continuous function with compact support, then

$$
\Psi(\mu * \chi) \geqq \Psi(\mu) * \chi
$$

on any open set $\Omega^{\prime}$ with $\Omega^{\prime}+$ support $\chi \subset \Omega$.

To prove Proposition 5.4, it is convenient to use an alternate definition of $\Psi(\mu)$.

Lemma 5.5. For all Borel subsets $E$ of $\Omega$,

$$
\Psi(\mu)(E)=\inf \left\{\sum_{j=1}^{\infty} \Psi\left(\mu\left(E_{j}\right)\right): E=\bigcup_{j=1}^{\infty} E_{j}, E_{j} \text { disjoint Borel subsets of } \Omega\right\} .
$$

Proof. This is a special case of [12], Theorem 2, p. 163, with only three slight modifications. First, Goffman and Serrin treat subadditive functions, while $\Psi$ 
is superadditive. However, the same arguments will work in our case. Second, Goffman and Serrin take the infinimum as the definition of $\Psi(\mu)(E)$, and then prove our definition coincides with the infinimum definition. Third, Goffman and Serrin assume that the domain of $\Psi$ is all of Euclidean space (or a Banach space) instead of just a cone. However, this is not an essential change.

Proof of Proposition 5.4. If $E=\bigcup E_{j}$, we have

$$
\Psi\left((\mu * \chi)\left(E_{j}\right)\right)=\Psi\left(\int \chi(x) \mu\left(E_{j}-\{x\}\right) d x\right) \geqq \int \chi(x) \Psi\left(\mu\left(E_{j}-\{x\}\right)\right) d x,
$$

where the last inequality results from the homogenity of $\Psi$ and Jensen's inequality for concave functions. Summing over $j$ gives

$$
\begin{aligned}
\sum_{j} \Psi\left((\mu * \chi)\left(E_{j}\right)\right) & \geqq \int \chi(x) \sum_{j} \Psi\left(\mu\left(E_{j}-\{x\}\right)\right) d x \\
& \geqq \int \chi(x) \Psi(\mu)(E-\{x\}) d x=(\Psi(\mu) * \chi)(E)
\end{aligned}
$$

where the last inequality follows from Lemma 5.5. Taking the infinimum over all Borel partitions $E=\bigcup E_{j}$ of $E$ and applying Lemma 5.5 again, the Proposition follows.

The other fact we need about the operator $\Psi$ is a semicontinuity property.

Proposition 5.6. Let $\left\{\mu^{j}\right\}$ be a sequence of Borel measures on $\Omega$ with values in $C$ which converges weakly to the Borel measure $\mu$. Suppose also that the Borel measures $\Psi\left(\mu^{j}\right)$ converge weakly. Then

$$
\Psi(\mu) \geqq \lim _{j \rightarrow \infty} \Psi\left(\mu^{j}\right) .
$$

Proof. This is a special case of [12], Theorem 3, p. 165, with the same slight modifications observed in Lemma 5.5 .

We now define the operator $\Phi(u)$. If $u$ is plurisubharmonic on $\Omega$, then the matrix of Borel measures $\left(\frac{\partial^{2} u}{\partial z_{j} \partial \bar{z}_{k}}\right)$ takes values in the cone $C$ since $d d^{c} u$ is a
positive $(1,1)$ current. Definition. $\Phi(u)=4(n !)^{1 / n} \Psi\left(\left[\frac{\partial^{2} u}{\partial z_{j} \partial \bar{z}_{k}}\right]\right)$.

We can now very easily give several properties of $\Phi(u)$.

Theorem 5.7. Let $u, v, u_{j}$ be plurisubharmonic on $\Omega \subset \mathbb{C}^{n}$. Then

(1) $\Phi(\alpha u)=\alpha \Phi(u), \quad \alpha \geqq 0$;

(2) $\Phi(t u+(1-t) v) \geqq t \Phi(u)+(1-t) \Phi(v), \quad 0<t<1$;

(3) If $\chi \geqq 0$ is a continuous function with compact support, then

$\Phi(u * \chi) \geqq \Phi(u) * \chi$

for any open set $\Omega^{\prime}$ with $\Omega^{\prime}+$ support $\chi \subset \Omega$;

(4) if $u_{j} \rightarrow u$ locally in $L^{1}$, or as distributions on $\Omega$, and if the sequence of measures $\Phi\left(u_{j}\right)$ converges weakly, then

$\Phi(u) \geqq \lim _{j \rightarrow \infty} \Phi\left(u_{j}\right) ;$ 
(5) If $u_{\varepsilon}=u * \chi_{\varepsilon}$, where $\chi_{\varepsilon}(z)=\frac{1}{\varepsilon^{2 n}} \chi\left(\frac{z}{\varepsilon}\right) \geqq 0$ is a usual smoothing kernel (e.g. [13], p. 45), then

$\lim _{\varepsilon \rightarrow 0} \Phi\left(u_{\varepsilon}\right)=\Phi(u)$.

(6) $\Phi(\max (u, v)) \geqq \min (\Phi(u), \Phi(v))$.

Proof. Assertions (1), (2) follow from Proposition 5.3. Assertion 3 follows directly from Proposition 5.4. To see that (4) follows from Proposition 5.6, note that the operator $d d^{c}$ is continuous for the distribution topology, so $d d^{c} u_{j} \rightarrow d d^{c} u$ as $(1,1)$ currents on $\Omega$. However, just as convergence of positive measures in the distribution topology implies weak convergence as Borel measures on $\Omega$, we have that $d d^{c} u_{j} \rightarrow$ $d d^{c} u$ as Borel measures, i.e. $\int \phi \wedge d d^{c} u_{j} \rightarrow \int \phi \wedge d d^{c} u$ for all $(n-1, n-1)$ forms $\phi$ with coefficients which are continuous and have compact support. (See e.g. [17], p. 67.) Thus, Proposition 5.6 implies assertion (4).

To prove (5), we combine (3) and (4). That is, we have $\Phi\left(u_{\varepsilon}\right) \geqq \Phi(u) * \chi_{\varepsilon}$ by (3), and by (4), as $\varepsilon \rightarrow 0$, we have

$$
\Phi(u) \geqq \lim _{\varepsilon \rightarrow 0} \Phi\left(u_{\varepsilon}\right) \geqq \lim _{\varepsilon \rightarrow 0} \Phi(u) * \chi_{\varepsilon}=\Phi(u)
$$

where the last equality is a well-known property of the smoothing kernels $\chi_{\varepsilon}$.

To prove (6), first assume $u, v$ are smooth. Then on the open set where $u>v$, we have

$$
\Phi(\max (u, v))=\Phi(u) \geqq \min (\Phi(u), \Phi(v)),
$$

and a similar inequality where $v>u$. Thus, unless $u=v$ on a set of positive measure, this case is proved. If $u=v$ on a set of positive measure, replace $v$ by $v+\varepsilon$. For all except countably many $\varepsilon>0$, the set $u=v+\varepsilon$ has zero measure. Thus,

$$
\Phi(\max (u, v+\varepsilon)) \geqq \min [\Phi(u), \Phi(v+\varepsilon)]=\min [\Phi(u), \Phi(v)] .
$$

Now let $\varepsilon \rightarrow 0$, and apply (4), to get assertion (6) when $u, v$ are smooth.

In the general case, replace $u, v$ by $u_{\varepsilon}, v_{\varepsilon}$. Then $\max \left(u_{\varepsilon}, v_{\varepsilon}\right)$ decreases to $\max (u, v)$ $\left([13]\right.$, p. 45). Select a sequence $\varepsilon_{j} \rightarrow 0$ such that $\Phi\left(\max \left(u_{\varepsilon_{j}}, v_{\varepsilon_{j}}\right)\right), \Phi\left(v_{\varepsilon_{j}}\right) \Phi\left(v_{\varepsilon_{j}}\right)$ and $\min \left[\Phi\left(u_{\varepsilon_{j}}\right), \Phi\left(v_{\varepsilon_{j}}\right)\right]$ all converge weakly. Then by (4) and (3),

$$
\begin{aligned}
\Phi(\max (u, v)) & \geqq \lim _{j \rightarrow \infty} \Phi\left(\max \left(u_{\varepsilon_{j}}, v_{\varepsilon_{j}}\right)\right) \\
& \left.\geqq \lim _{j \rightarrow \infty} \min \left(\Phi\left(u_{\varepsilon_{j}}\right)\right), \Phi\left(v_{\varepsilon_{j}}\right)\right) \\
& \geqq \lim _{j} \min \left[\Phi(u) * \chi_{\varepsilon_{j}}, \Phi(v) * \chi_{\varepsilon_{j}}\right] \\
& \geqq \lim _{j \rightarrow \infty} \min [\Phi(u), \Phi(v)] * \chi_{\varepsilon_{j}}=\min [\Phi(u), \Phi(v)] .
\end{aligned}
$$

This completes the proof.

We conclude this section by giving some explanation of the relationship between $\Phi(u)$ and $\left(d d^{c} u\right)^{n}$.

Theorem 5.8. Let $u$ be a plurisubharmonic function on $\Phi$ such that the regularizations of $u, u_{\varepsilon}=u * \chi_{\varepsilon}$, have the property

$\left\{\left(d d^{c} u_{\varepsilon}\right)^{n}\right\}$ is a bounded family of Borel measures on each compact subset of $\Omega$. 
Then

(1) $\Phi(u)$ is absolutely continuous with respect to Lebesgue measure, and, if $\Phi(u)=g d V$, where $d V$ is Lebesgue measure, then $g$ is locally $n$-th power integrable; i.e. $g \in L_{\text {loc }}^{n}(\Omega)$.

(2) if $u$ is continuous, and if $\left(d d^{c} u\right)^{n}=f d V+d v$ is the Lebesgue decomposition of the nonnegative measure $\left(d d^{c} u\right)^{n}$ into its absolutely continuous and singular parts, then

$g \leqq f^{1 / n}$

(3) if $\frac{\partial^{2} u}{\partial z_{j} \partial \bar{z}_{k}}=f_{j k} d V+d v_{j k}$ is the Lebesgue decomposition of the Borel measures $\frac{\partial^{2} u}{\partial z_{j} \partial \bar{z}_{k}}$, then $g=4(n !)^{1 / n} \operatorname{det}\left[f_{j k}\right]^{1 / n}$.

Proof. Since $u_{\varepsilon}$ is smooth, we can write $\Phi\left(u_{\varepsilon}\right)=g_{\varepsilon} d V$, and from (2.1),

$g_{\varepsilon}^{n} d V=\left(d d^{c} u_{\varepsilon}\right)^{n}$

Thus, from (5.5) we find that $\int_{K} g_{\varepsilon}^{n} d V \leqq M<\infty$ for each compact subset $K$ of $\Omega$. Thus, the $\left\{g_{\varepsilon}\right\}$ are a bounded family in $L_{\text {loc }}^{n}(\Omega)$. The measures $g_{\varepsilon} d V$ therefore have a subsequence which converges to a measure $g d V$, with $g \in L_{\text {loc }}^{n}(\Omega)$. But we already know by (5) of Theorem 5.7 that $g_{\varepsilon} d V \rightarrow \Phi(u)$. Thus, $\Phi(u)=g d V$. This proves (1).

To prove (2), note first that $\left(g * \chi_{\varepsilon}\right) d V \leqq \Phi\left(u_{\varepsilon}\right)$, by (3) of Theorem 5.7. Thus, from Fatou's lemma we find for all $\phi \geqq 0$, continuous with compact support in $\Omega$,

$$
\begin{aligned}
\int \phi g^{n} d V & \leqq \liminf _{\varepsilon \rightarrow 0} \int \phi\left(g * \chi_{\varepsilon}\right)^{n} d V \\
& \leqq \liminf _{\varepsilon \rightarrow 0} \int \phi\left(g_{\varepsilon}\right)^{n} d V \\
& =\lim _{\varepsilon \rightarrow 0} \int \phi\left(d d^{c} u_{\varepsilon}\right)^{n} \\
& =\int \phi f d V+\int \phi d v .
\end{aligned}
$$

Thus, $g^{n} d V \leqq f d V+d v$, so $g^{n} \leqq f$.

To prove (3), we use (2) of Proposition 5.3. Thus, $\left.\frac{\Phi(u)}{4(n !)^{1 / n}}=\Psi\left(\left[f_{j k} d V\right]\right)+\Psi\left[d v_{j k}\right]\right)$. However, by (1), $\Phi(u)$ is absolutely continuous, and by (3) of Proposition 5.3, $\Psi\left[d v_{j k}\right]$ is singular with respect to Lebesgue measure. Thus $\Psi\left[d v_{j k}\right]=0$. This completes the proof.

In connection with Theorem 5.8, we mention the following example. If $u=\log |z|$, then $\Phi(u) \equiv 0$, as a short computation shows. Thus, there can be no minimum principle for the operator $\Phi$ analogous to Theorem 2 .

Finally, we know of no example where the measure $\Phi(u)$ is not absolutely continuous with respect to Lebesgue measure.

\section{Regularity Properties of Upper Envelopes}

Now we shall define some classes of plurisubharmonic functions which are subsolutions of the Dirichlet problem (1.1). In Section 8 it will be shown that the 
solution to (1.1) may be obtained as the supremum taken over these classes of subsolutions. In this section we show that these upper envelopes have desirable regularity properties. First, the upper envelope is shown to lie in Lip ${ }^{\alpha}$ if the data are sufficiently regular (Theorem 6.2). Next an approximation to the Laplacian is introduced which behaves well with respect to the families of subsolutions. In particular, it is shown (Corollary 6.5) that if $u \in P(\Omega) \cap C^{2}(\bar{\Omega})$ and $\Phi(u) \in P(\Omega)$ then $\frac{\partial^{2} u}{\partial z_{j} \partial \bar{z}_{j}}$ assumes its maximum on $\partial \Omega$. Finally, it will be shown (Theorem 6.9) that if $\mu$ and $\phi$ are of class $C^{2}$, and if $\Omega=B^{n}$ is the unit ball in $\mathbb{C}^{n}$, then the upper envelope of subsolutions actually has locally bounded second derivatives. This result is obtained by using the group of analytic automorphisms of $B^{n}$.

Given $\phi \in C(\partial \Omega)$ and a measure $\mu$ on $\Omega$, we define three Perron-Bremermann families of subsolutions to (1.1) for the operators $\Phi$ and $\left(d d^{c}\right)^{n}$.

$$
\begin{aligned}
& \mathscr{B}(\phi, \mu)=\left\{v \in P(\Omega): \Phi(v) \geqq \mu \text { and } \limsup _{z \rightarrow z_{0}} v(z) \leqq \phi\left(z_{0}\right), \text { for all } z_{0} \in \partial \Omega\right\} . \\
& C \mathscr{B}(\phi, \mu)=\mathscr{B}(\phi, \mu) \cap C(\bar{\Omega}),
\end{aligned}
$$

and

$$
\mathscr{F}(\phi, \mu)=\left\{v \in P(\Omega) \cap C(\bar{\Omega}):\left(d d^{c} v\right)^{n} \geqq \mu \text { and } v\left(z_{0}\right) \leqq \phi\left(z_{0}\right) \text { for all } z_{0} \in \partial \Omega\right\} .
$$

Throughout the remainder of this section the domain $\Omega$ is assumed to be strictly pseudoconvex.

If $\mu \in L_{\text {loc }}^{n}(\Omega)$, say $\mu=f d V$ ( $d V=$ Lebesgue measure), then let $\mu^{n}=f^{n} d V$. If $v \in P(\Omega) \cap C(\Omega)$, then by Theorem 5.8 ,

$$
\Phi(v)^{n} \leqq\left(d d^{c} v\right)^{n}
$$

and consequently,

$$
\operatorname{C\mathscr {B}}(\phi, \mu) \subseteq \mathscr{F}\left(\phi, \mu^{n}\right) \text {. }
$$

Theorem 6.2 will show that if, in addition, $f \in C(\bar{\Omega})$, then $u(z)=\sup \{v(z): v \in \mathscr{B}(\phi, \mu)\}$ belongs to $C(\bar{\Omega}) \cap P(\Omega)$, which will allow us to conclude

$$
\sup \{v: v \in \mathscr{B}(\phi, \mu)\}=\sup \{v: \in C \mathscr{B}(\phi, \mu)\} \leqq \sup \left\{v: v \in \mathscr{F}\left(\phi, \mu^{n}\right)\right\} \text {. }
$$

In Theorem 6.9, these three envelopes will be shown to be the same if $\Omega$ is the unit ball, and then in Section 8 it will be established that these envelopes coincide and solve the Dirichlet problem (1.1) for all strictly pseudoconvex sets $\Omega$ when $\phi$ and $d \mu=f d V$ are continuous.

Note that in case the measure $\mu$ is not absolutely continuous with respect to $d V$, the class $C \mathscr{B}(\phi, \mu)$ is empty, by Theorem 5.8, part (1).

The following proposition justifies the terminology "subsolution" for the families $C \mathscr{B}$ and $\mathscr{F}$.

Proposition 6.1. Let $\Omega$ be a bounded domain in $\mathbb{C}^{n}$ and suppose $u \in P(\Omega) \cap C(\bar{\Omega})$ satisfies $\left(d d^{c} u\right)^{n}=(\Phi(u))^{n}$. If $C \mathscr{B}=C \mathscr{B}(\phi, \Phi(u))$ and $\mathscr{F}=\mathscr{F}\left(\phi,\left(d d^{c} u\right)^{n}\right)$, where $\phi=\left.u\right|_{\partial \Omega}$, then $\sup \{v: v \in \mathscr{F}\}=\sup \{v: v \in C \mathscr{B}\}=u$.

Proof. From Theorem 5.8, part (1), we deduce that

$$
u \leqq \sup \{v: v \in C \mathscr{B}\} \leqq \sup \{v: c \in \mathscr{F}\}
$$


so it suffices to show that

$\sup \{v: v \in \mathscr{F}\} \leqq u$.

However, if $v \in \mathscr{F}$, then by Theorem A, $u-v$ attains its minimum on $\bar{\Omega}$ on the boundary of $\Omega$. Thus, since $u \geqq v$ on $\partial \Omega$, we have $u \geqq v$ in $\Omega$ for all $v \in \mathscr{F}$, and the proposition follows.

We next discuss Lipschitz regularity of the envelope. By $\operatorname{Lip}^{\alpha}(X), 0<\alpha \leqq 1$, we mean the functions on $X$ which satisfy a Lipschitz condition of order $\alpha$,

$$
|u(z)-u(w)| \leqq C|z-w|^{\alpha}
$$

If $\alpha=0$, $\operatorname{Lip}^{0}(X)=C(X)$, the continuous functions on $X$. If $\alpha>1$, say $k<\alpha \leqq k+1$, then by $\operatorname{Lip}^{\alpha}(X)$ we mean the class of functions which have continuous partial derivatives of order $\leqq k$ on $X$, and whose $k$-th order partial derivatives satisfy a Lipschitz condition of order $\alpha-k$.

The following proof is modeled on a Proposition of J.B. Walsh [23].

Theorem 6.2. Let $\Omega$ be a strongly pseudoconvex, bounded domain in $\mathbb{C}^{n}$ with $C^{2}$ boundary. Suppose that for some $\alpha, 0 \leqq \alpha \leqq 1$, we have $\phi \in \operatorname{Lip}^{2 \alpha}(\partial \Omega)$ and $d \mu=f d V$ with $f \in \operatorname{Lip}^{\alpha}(\bar{\Omega})$. Then the upper envelope $u$ of $\mathscr{B}(\phi, \mu)$,

$$
u(z)=\sup \{v(z): v \in \mathscr{B}(\phi, \mu)\}
$$

belongs to $\operatorname{Lip}^{\alpha}(\bar{\Omega})$ and is plurisubharmonic on $\Omega$.

Proof. The upper envelope of a family of plurisubharmonic functions is plurisubharmonic if it is continuous, so we only have to show $u \in \operatorname{Lip}^{\alpha}(\bar{\Omega})$. We will first prove that given $\zeta \in \partial \Omega$, there exists $v \in \mathscr{B}(\phi, \mu) \cap \operatorname{Lip}^{\alpha}(\bar{\Omega})$ such that $v(\zeta)=\phi(\zeta)$. We do this assuming $\alpha>0$, since the case $\alpha=0$ (i.e. $\phi, \mu$ continuous) was treated by Bremermann ([4], p. 250). It is sufficient to prove that there exists a constant $C$, depending only on $\Omega$, such that for all $\zeta \in \partial \Omega$, and all $\phi \in \operatorname{Lip}^{2 \alpha}(\partial \Omega)$, there exists a function $h_{\zeta} \in \operatorname{Lip}^{\alpha}(\bar{\Omega}) \cap P(\Omega)$ such that

$$
\begin{aligned}
& h_{\zeta}(z) \leqq \phi(z), \quad z \in \partial \Omega \\
& h_{\zeta}(\zeta)=\phi(\zeta), \\
& \left\|h_{\zeta}\right\|_{\alpha} \leqq C\|\phi\|_{2 \alpha}
\end{aligned}
$$

where by $\|h\|_{\alpha},\|\phi\|_{\alpha}$ we mean any convenient norms on the Banach spaces $\operatorname{Lip}^{\alpha}(\bar{\Omega}), \operatorname{Lip}^{\alpha}(\partial \Omega)$, e.g. if $0<\alpha \leqq 1$,

$$
\|h\|_{\alpha}=\sup \{|h(z)|: z \in \bar{\Omega}\}+\sup \left\{\frac{|h(z)-h(w)|}{|z-w|^{\alpha}}: z, w \in \bar{\Omega}\right\} .
$$

Because, if (6.1)-(6.3) are proved, then we first choose $K_{1}$ so large that $\Phi\left(K_{1}|z|^{2}\right)$ $=K_{1} \Phi\left(|z|^{2}\right) \geqq \mu$ and then $K_{2}=K_{1}|\zeta|^{2}, \tilde{\phi}(z)=\phi(z)-K_{1}|z|^{2}+K_{2}$ and the function $h=h_{\zeta}$ so that (6.1)-(6.3) hold with $\phi$ replaced by $\tilde{\phi}$. The function $v \in \mathscr{B}(\phi, \mu) \cap \operatorname{Lip}^{\alpha}(\bar{\Omega})$ is then

$$
v(z)=h(z)+K_{1}|z|^{2}-K_{2} .
$$

We also obtain a bound

$$
\|v\|_{\alpha} \leqq C\left(\|\phi\|_{2 \alpha}+K_{1}\right)
$$

for some constant $C$ (different from the constant of (6.3)). 
To prove (6.1)-(6.3), make a holomorphic change of coordinates so that the boundary is given by $x_{1}=y_{1}^{2}+\left|z_{2}\right|^{2}+\cdots+\left|z_{n}\right|^{2}+\sigma(z)$, where $\sigma(z)=o\left(|z|^{2}\right)$ and $\zeta$ is the origin of coordinates $\left(z_{1}, \ldots, z_{n}\right)$. We may assume that $\phi(0)=0$ and, if $2 \alpha-1>0, d \phi(0)=0$. Thus, for $z \in \partial \Omega, z$ near $0, \phi(z) \geqq-c|z|^{2 \alpha}$, where $c>0$. Set $h(z)=-2 c\left|x_{1}\right|^{\alpha}$. Observe that, since $0 \leqq \alpha \leqq 1, h$ is plurisubharmonic and that for $z \in \partial \Omega$ near 0 ,

$$
\tilde{h}(z)=-2 c\left(y_{1}^{2}+\left|z_{2}\right|^{2}+\cdots+\left|z_{n}\right|^{2}+\sigma(z)\right)^{\alpha} \leqq-c|z|^{2 \alpha} \leqq \phi(z)
$$

while $\tilde{h}(0)=0=\phi(0)$. Now $\tilde{h}$, via the inverse change of coordinates, is given as a function $h$ plurisubharmonic near $\zeta \in \partial \Omega$. The norm $\|h\|_{\alpha}$ is determined by the mapping function giving the analytic change of coordinates. Further, by choosing a function

$$
h_{\zeta}(z)=\max \left(-\lambda_{1}, \lambda_{2} h(z)\right)
$$

where $\lambda_{2} \geqq 1$ is large and $\lambda_{1}>0$ is small, we obtain properties (6.1), (6.2), (6.3). The constant $C$ in (6.3) is seen to depend only on $\Omega$.

Next, we assert that there exists $v \in \mathscr{B}(\phi, \mu) \cap \operatorname{Lip}^{\alpha}(\bar{\Omega})$ such that $v(\zeta)=\phi(\zeta)$ for all $\zeta \in \partial \Omega$. We have just proved that for each $\zeta \in \partial \Omega$, there is a function $v_{\zeta} \in \mathscr{B}(\phi, \mu)$ with $v_{\zeta}(\zeta)=\phi(\zeta)$ and $\left\|v_{\zeta}\right\|_{\alpha} \leqq C$ for some constant $C$ independent of $\zeta$. Set

$$
v(z)=\sup \left\{v_{\zeta}(z): \zeta \in \partial \Omega\right\} \text {. }
$$

Then from $\left|v_{\zeta}(z)-v_{\zeta}(w)\right| \leqq C|z-w|^{\alpha}$, we deduce that $|v(z)-v(w)| \leqq C|z-w|^{\alpha}$, so $v \in \operatorname{Lip}^{\alpha}(\bar{\Omega}) \cap P(\Omega)$, and clearly $v(\zeta)=\phi(\zeta)$ for all $\zeta \in \partial \Omega$. It then follows from (6) and (4) of Theorem 5.7 that also $\Phi(v) \geqq \mu$, so $v \in \mathscr{B}(\phi, \mu) \cap \operatorname{Lip}^{\alpha}(\bar{\Omega})$, as asserted.

By a similar construction there exists a plurisuperharmonic function $w \in \operatorname{Lip}^{\alpha}(\bar{\Omega})$ such that $w(z)=\phi(z)$ for $z \in \Omega$. Thus for $z \in \Omega$

$$
\begin{aligned}
& v(z) \leqq u(z) \leqq w(z), \quad \text { and so if } z \in \Omega, \quad \zeta \in \partial \Omega, \\
& |u(z)-u(\zeta)| \leqq K|z-\zeta|^{\alpha} .
\end{aligned}
$$

Now we must show that (6.5) also holds for $\zeta \in \Omega$.

Let $u^{*}(z)=\limsup _{\Delta z \rightarrow 0} u(z+\Delta z)$ be the upper regularization of $u$. Then $u^{*}$ is plurisubharmonic ([17], p. 26). Because of (6) of Theorem 5.7, $\mathscr{B}(\phi, \mu)$ is closed under the operation of taking finite maxima. Thus, we can choose a sequence $u_{j} \in \mathscr{B}(\phi, \mu)$ with $u_{1} \leqq u_{2} \leqq \ldots$ and $u_{j} \rightarrow u$ almost everywhere. Since $u=u^{*}$ almost everywhere, we have that $u_{j} \rightarrow u^{*}$ locally in $L^{1}$, so that $d d^{c} u_{j}$ converges weakly to $d d^{c} u^{*}$. From (4) of Theorem 5.7, it follows that

$$
\Phi\left(u^{*}\right) \geqq \lim _{j \rightarrow \infty} \Phi\left(u_{j}\right) \geqq \mu .
$$

Then, by (6.4), $u^{*} \in \mathscr{B}(\phi, \mu)$. Since $u \leqq u^{*}$, we must have $u=u^{*}$, so $u$ is plurisubharmonic and $u \in \mathscr{B}(\phi, \mu)$.

For any small vector $\tau \in \mathbb{C}^{n}$, define

$$
V(z, \tau)= \begin{cases}u(z) & z+\tau \notin \Omega, z \in \bar{\Omega}, \\ \max (u(z), v(z)), & z, z+\tau \in \Omega\end{cases}
$$

where

$$
v(z)=u(z+\tau)+K_{3}|\tau|^{\alpha}|z|^{2}-K_{4}|\tau|^{\alpha}-K_{5}|\tau|^{\alpha}
$$


and

$$
K_{3}=\|\mu\|_{\alpha}=\|f\|_{\alpha}, \quad K_{4}>K_{3}|\tau|^{\alpha}|z|^{2}
$$

for all $z \in \Omega$, and $K_{5}$ is the constant $K$ of (6.5). Observe that for all $\tau, z \rightarrow V(z, \tau) \in P(\Omega)$. For, by (6.5), $v(z)<u(z)$ if $z \in \Omega, z+\tau \in \partial \Omega$. From the choice of $K_{3}$ and $K_{4}$, we have $V(z, \tau) \in \mathscr{B}(\phi, \mu)$. It follows that for all $z \in \Omega, V(z, \tau) \leqq u(z)$. If $z+\tau \in \bar{\Omega}$, this yields

$$
u(z+\tau)-u(z) \leqq K|\tau|^{\alpha}
$$

for a suitable constant $K$. Reversing the roles of $z+\tau$ and $z$, we obtain

$$
|u(z+\tau)-u(z)| \leqq K|\tau|^{\alpha},
$$

whence $u \in \operatorname{Lip}^{\alpha}(\bar{\Omega})$. If $\alpha=0$, only minor modifications need be made in the argument to show that $u$ is continuous. This completes the proof.

Remark. If $\phi \in \operatorname{Lip}^{2 \alpha}(\partial \Omega), \mu \in C^{\infty}$, then in general $u \notin \operatorname{Lip}^{\alpha+\varepsilon}$ for $\varepsilon>0$. This is easily seen for the real Monge-Ampere equation in the unit disc in $\mathbb{R}^{2}$ with $\phi(\theta)=\theta^{2 \alpha}$ and $\mu \equiv 0$.

For any $u \in C(\Omega)$ and any vector $\zeta \in \mathbb{C}^{n},|\zeta|=1$, define

$$
u_{\varepsilon}(z)=u_{\zeta, \varepsilon}(z)=\frac{1}{2 \pi} \int_{0}^{2 \pi} u\left(z+\varepsilon e^{i \theta} \zeta\right) d \theta
$$

and

$$
T_{\zeta, \varepsilon} u(z)=\varepsilon^{-2}\left[u_{\zeta, \varepsilon}(z)-u(z)\right] .
$$

Further, let

$$
\frac{\partial^{2} u(z)}{\partial \zeta \partial \bar{\zeta}^{\prime}}=\sum_{i, j=1}^{n} \frac{\partial^{2} u(z)}{\partial z_{i} \partial \bar{z}_{j}} \zeta_{i} \bar{\zeta}_{j}
$$

Proposition 6.3. For $u \in C(\Omega)$,

$$
\lim _{\varepsilon \rightarrow 0} T_{\zeta, \varepsilon} u=\frac{\partial^{2} u}{\partial \zeta \partial \bar{\zeta}}
$$

in the following senses:

(1) if $u \in C^{2}(\Omega)$, the limit exists in $C(\Omega)$;

(2) if $u \in C(\Omega)$, the limit exists in $\mathscr{D}^{\prime}(\Omega)$;

(3) If $u \in P(\Omega)$, the limit exists in the sense of weak convergence of measures;

(4) if $u \in L_{2}^{\infty}(\Omega)$ (i.e. has second partial derivatives in $L^{\infty}(\Omega)$ ), then the limit exists weakly in $L_{\mathrm{loc}}^{\infty}(\Omega)$.

Proof. These facts are all well-known. We will only outline the proofs. Statement (1) may be seen if $u$ is written as a Taylor polynomial with remainder. Then (2) follows from (1), since for all $\phi \in \mathscr{D}(\Omega)$,

$$
\left(T_{\zeta, \varepsilon} u, \phi\right)=\int_{\Omega} T_{\zeta, \varepsilon} u(z) \phi(z) d V(z)=\int_{\Omega} u(z)\left(T_{\zeta, \varepsilon} \phi\right)(z) d V(z)=\left(u, T_{\zeta, \varepsilon} \phi\right) \rightarrow\left(u, \Delta_{\zeta} \phi\right) .
$$


To establish (3) it is sufficient to show that the $L^{1}$-norms of $T_{\zeta, \varepsilon} u$ remain bounded as $\varepsilon \rightarrow 0$. If $u \in P(\Omega)$ it follows from Jensen's formula in one variable that

$$
\int_{|z| \leqq r} T_{\zeta, \varepsilon} u(z) d V(z) \leqq \int_{|z| \leqq r+\varepsilon} \frac{\partial^{2} u}{\partial \zeta \partial \bar{\zeta}}(z) d V(z)
$$
and so $T_{\zeta, \varepsilon} u$ has a weak limit in the space of measures, which must be $\frac{\partial^{2} u}{\partial \zeta \partial \zeta}$ by (2).
The statement (4) follows in a similar way from Jensen's inequality.

Theorem 6.4. Let $\Omega$ be a bounded open set in $\mathbb{C}^{n}$. Suppose that $u \in P(\Omega) \cap C(\bar{\Omega})$ and that

$$
\left(d d^{c} u\right)^{n}=f d V, \quad \Phi(u)=f^{1 / n} d V
$$

where $d V$ is Lebesgue measure, $0 \leqq f \in L_{\mathrm{loc}}^{1}(\Omega)$, and $f^{1 / n} \in P(\Omega)$. Then for $\varepsilon>0$ and $\zeta \in \mathbb{C}^{n},|\zeta|=1$, the function $T_{\zeta, \varepsilon} u$ is defined on the closure of $\Omega^{\varepsilon}=\{z \in \Omega$ : dist. $(z, \partial \Omega)>\varepsilon\}$ and

$$
\sup \left\{\left(T_{\zeta, \varepsilon} u\right)(z): z \in \Omega^{\varepsilon}\right\}=\sup \left\{\left(T_{\zeta, \varepsilon} u\right)(z): z \in \partial \Omega^{\varepsilon}\right\} .
$$

Proof. It is clear that $T_{\zeta, \varepsilon} u=T_{\varepsilon} u$ is defined on the closure of $\Omega^{\varepsilon}$. Suppose that $T_{\varepsilon} u$ has a strict interior maximum, i.e. for some $\eta>0$ and some $z_{0} \in \Omega^{\varepsilon}$,

$$
T_{\varepsilon} u\left(z_{0}\right)-\eta>\sup _{z \in \partial \Omega^{\varepsilon}} T_{\varepsilon} u(z)=C .
$$

Then we may define the function

$$
v(z)=u_{\varepsilon}(z)-\varepsilon^{2}(C+\eta)
$$

By Theorem 5.7 and the fact that $\Phi(u) \in P(\Omega)$,

$$
\Phi(v) \geqq \Phi(u)_{\varepsilon} \geqq \Phi(u) \text {. }
$$

Set $\phi^{\varepsilon}=\left.u\right|_{\partial \Omega^{\varepsilon}}$. Then we have

$$
v \in C \mathscr{B}\left(\phi^{\varepsilon},[\Phi(u)]^{n}, \Omega^{\varepsilon}\right),
$$

since for $z \in \partial \Omega^{\mathrm{r}}$,

$$
\begin{aligned}
v(z) & =u_{\varepsilon}(z)-\varepsilon^{2}(C+\eta) \\
& \leqq u_{\varepsilon}(z)-\varepsilon^{2} \eta-\varepsilon^{2} T_{\varepsilon} u(z)<u(z)=\phi^{\varepsilon}(z) .
\end{aligned}
$$

On the other hand,

$$
\begin{aligned}
v\left(z_{0}\right) & =u_{\varepsilon}\left(z_{0}\right)-\varepsilon^{2}(C+\eta) \\
& >u_{\varepsilon}\left(z_{0}\right)-\varepsilon^{2}\left(T_{\varepsilon} u\left(z_{0}\right)\right)=u\left(z_{0}\right),
\end{aligned}
$$

which contradicts Proposition 6.1.

We can now prove Theorem B by the same method.

Proof of Theorem B. Define

$$
H(a, z)=\sum_{j, k=1}^{n} \frac{\partial^{2} u}{\partial z_{j} \partial \bar{z}_{k}}(z) a_{j k}, \quad z \in \bar{\Omega} .
$$


After a unitary change of coordinates, the expression for $H(a, z)$ becomes

$$
\tilde{H}(a, z)=\sum_{j=1}^{n} \lambda_{j} \frac{\partial^{2} u}{\partial z_{j} \partial \bar{z}_{j}}(z)
$$

where the $\lambda_{j} \geqq 0$ are the eigenvalues of $a=\left(a_{j k}\right)$. Setting $e_{j}=(0,0, \ldots, 1,0, \ldots, 0)$, we see from (1) of Proposition 6.3 that

$$
\tilde{T}_{\varepsilon} u=\sum_{j=1}^{n} \lambda_{j} T_{e_{j}, \varepsilon} u
$$

converges to $\tilde{H}(a, z)$ uniformly on $\bar{\Omega}$ as $\varepsilon \rightarrow 0$, since $u \varepsilon C^{2}(\bar{\Omega})$. Therefore, if $\tilde{H}(a, z)$ has a strict interior maximum in $\bar{\Omega}$, then $\tilde{T}_{\varepsilon} u$ also has a strict interior maximum in $\Omega_{\varepsilon}$ when $\varepsilon$ is sufficiently small. However, the proof of Theorem 6.4 , with $T_{\zeta, \varepsilon}$ replaced by $\tilde{T}_{\varepsilon}$ and $u_{\varepsilon}$ replaced by $\sum_{j=1}^{n} \lambda_{j} u_{e_{j}, \varepsilon}$ shows that $\tilde{T}_{\varepsilon} u$ cannot have a strict interior maximum. Thus, $\tilde{H}(a, z)$ and $H(a, z)$ must attain their maximum on $\partial \Omega$.

Corollary 6.5. If $u$ satisfies the hypotheses of Theorem 6.4 and for some $\lambda>0$,

ess sup $\{\Delta u(z): z \in \Omega, \operatorname{dist}(z, \partial \Omega)<\lambda\}<\infty$, then $\|\Delta u\|_{L^{\infty}(\Omega)}<\infty$.

Proof. Since $\Delta u \in L^{\infty}$ near $\partial \Omega$, it follows that $\sum_{j=1}^{n} T_{e_{j}, \varepsilon} u$ converges weakly to $\frac{1}{4} \Delta u$ in $L^{\infty}(z \in \Omega$ : $\operatorname{dist}(z, \partial \Omega)<\lambda)$. Thus the set $\sum_{j=1}^{n} T_{e_{j}, \varepsilon} u$ is bounded on $\{z \in \Omega: \operatorname{dist}(z, \partial \Omega)<\lambda\}$ for $\varepsilon>0$ and by the proof of Theorem B, the same bound holds on all of $\Omega$. It follows that $\sum_{j=1}^{n} T_{e_{j}, \varepsilon} u$ converges weakly to $\frac{1}{4} \Delta u$ in $L^{\infty}(\Omega)$.

Proposition 6.6. Let $\Omega=B^{n}$ be the unit ball in $\mathbb{C}^{n}$. Suppose $\phi \in C^{2}\left(\partial B^{n}\right), d \mu=f d V$ with $f \in C^{2}\left(\overline{B^{n}}\right), f \geqq 0$. If

$$
u=\sup \{v: v \in \mathscr{B}(\phi, \mu)\}
$$

is the upper envelope of $\mathscr{B}(\phi, \mu)$, then for every $\eta>0$, there exists a constant $A(\eta)$ such that

$$
u(z+h)-2 u(z)+u(z-h) \leqq A(\eta)|h|^{2}
$$

for all $|z| \leqq 1-\eta$ and $|h| \leqq \eta / 2$.

Proof. For $a \in B^{n}$, let $T_{a} \in$ Aut $\left(B^{n}\right)$ be defined by

$$
T_{a}(z)=\Gamma(a) \frac{z-a}{1-\bar{a}^{t} z}
$$

where

$$
\Gamma(a)=\frac{a^{t} \bar{a}}{1-v(a)}-v(a) I
$$

and

$$
v(a)=\sqrt{1-|a|^{2}} .
$$


Here we are following the notation of [18], p. 6, and points of $\mathbb{C}^{n}$ are thought of as $n \times 1$ column matrices, so that $\Gamma(a)$ is an $n \times n$ matrix. Note that $T_{a}(a)=0$, $T_{-a}=T_{a}^{-1}$, and $T_{a}(z)$ is analytic in $z$, and a smooth function of $a \in B^{n}$.

For $a \in B(0,1-\eta)=\{a:|a|<1-\eta\}$, set

$$
L(a, h, z)=T_{a+h}^{-1} T_{a}(z)
$$

and

$$
\begin{aligned}
& U(a, h, z)=u(L(a, h, z)), \\
& F(a, h, z)=f(L(a, h, z)), \\
& \Psi(a, h, z)=\phi(L(a, h, z)), \quad z \in \partial B^{n} .
\end{aligned}
$$

It follows that $F \in C^{z}\left(B(0,1-\eta) \times B(0, \eta) \times \overline{B^{n}}\right)$. Similarly, since $U(a, h, z)=$ $\Psi(a, h, z)$ for $z \in \partial B^{n}$, we conclude that $U \in C^{2}\left(B(0,1-\eta) \times B(0, \eta) \times \partial B^{n}\right)$. Consequently, for a suitable constant $K_{1}$, depending on $\eta>0$, we have

$$
\frac{1}{2}(U(a, h, z)+U(a,-h, z))-K_{1}|h|^{2} \leqq U(a, 0, z)=\phi(z)
$$

for all $|a| \leqq 1-\eta,|h| \leqq \eta / 2$, and $z \in \partial B^{n}$. For example, take $K_{1}$ to be the supremum over the set $\overline{B(0,1-\eta)} \times \overline{B(0, \eta / 2)} \times \partial B^{n}$ of the norms of the second derivatives of $U$. If it can be shown that $z \rightarrow v(z) \in \mathscr{B}(\phi, \mu)$ where

$$
\begin{aligned}
v(a, h, z)= & \frac{1}{2}[U(a, h, z)+U(a,-h, z)]-K_{1}|h|^{2} \\
& +K_{2}\left(|z|^{2}-1\right)|h|^{2}
\end{aligned}
$$

then it follows that $v(a, h, z) \leqq u(z)$. Thus, if we set $a=z$, we conclude that

$$
\frac{1}{2}[u(z+h)+u(z-h)] \leqq u(z)+\left(K_{1}+K_{2}\right)|h|^{2}
$$

which proves the proposition.

Let $J L(a, h, z)$ be the Jacobian matrix of the holomorphic mapping $z \rightarrow L(a, h, z)$. Then, by the chain rule, we have

$$
\left[\frac{\partial^{2} U}{\partial z_{j} \partial \bar{z}_{k}}\right]=[J L(a, h, z)]^{t}\left[\frac{\partial^{2} u}{\partial z_{l} \partial \bar{z}_{m}}\right] \overline{[J L(a, h, z)]}
$$

where the multiplication on the right hand side is matrix multiplication. It then follows that

$$
\Phi(U)=\Phi(u)|\operatorname{det} J L(a, h, z)|^{2 / n}
$$

where $\Phi$ is applied to the plurisubharmonic function, $z \rightarrow U(a, h, z)$. By Theorem 5.7, $\Phi$ is superadditive (since $\Phi$ is concave and homogeneous of degree 1 ), so

$$
\Phi(v) \geqq \frac{1}{2}\left\{\Phi(U(a, h, z))+\Phi(U(a,-h, z)\}+4(n !)^{1 / n} K_{2}|h|^{2} d V\right.
$$

where the last term results from

$$
\Phi\left(|z|^{2}\right)=4(n !)^{1 / n} d V \text {. }
$$

Thus, by (6.9) and (6.10), if we want to show $v \in \mathscr{B}(\phi, \mu)$, it is sufficient to prove that for $K_{2}$ large enough,

$$
\frac{1}{2}\{F(a, h, z) j(a, h, z)+F(a,-h, z) j(a,-h, z)\}+K_{2}|h|^{2} \geqq F(a, 0, z)=f(z)
$$


for all $|a| \leqq 1-\eta,|h| \leqq \eta / 2$, and $z \in \overline{B^{n}}$, where $j(a, h, z)=|\operatorname{det} J L(a, h, z)|^{2 / n}$. However, $L(a, 0, z)$ is the identity mapping, so $j(a, 0, z) \equiv 1$. Since the function $G(a, h, z)=$ $F(a, h, z) j(a, h, z)$ is of class $C^{2}$ on a neighborhood of $|a| \leqq 1-\eta,|h| \leqq \eta / 2,|z| \leqq 1$, there exists $K_{2}$ such that

$$
\frac{1}{2}\{G(a, h, z)+G(a,-h, z)\}+K_{2}|h|^{2} \geqq G(a, 0, z)
$$

for $|a| \leqq 1-\eta,|h| \leqq \eta / 2,|z| \leqq 1$. This proves (6.11) and completes the proof of the proposition.

We can now prove a stronger regularity theorem, essentially Theorem C.

Theorem 6.7. Let $\Omega=B^{n}$ be the unit ball in $\mathbb{C}^{n}$. Let $\phi \in C^{2}\left(\partial B^{n}\right)$, and $d \mu=f d V$, with $f \in C^{2}\left(\overline{B^{n}}\right)$. If $u$ is the upper envelope of $\mathscr{B}(\phi, \mu)$.

$$
u(z)=\sup \{v(z): v \in \mathscr{B}(\phi, \mu)\}
$$

then $u \in L_{2,10 c}^{\infty}\left(B^{n}\right)$; i.e. $u$ has locally bounded second partial derivatives.

Proof. We will first show that for each $\zeta \in \mathbb{C}^{n},|\zeta|=1, \frac{\partial^{2} u}{\partial \zeta \partial \zeta} \in L_{2, \text { loc }}^{\infty}\left(B^{n}\right)$. To see this, we use the operators $T_{\zeta, \varepsilon}$ of (6.6). If $0<\eta<1$, and $|z| \leqq 1-\eta, \varepsilon \leqq \eta / 2$, then

$$
\begin{aligned}
\left(T_{\zeta, \varepsilon} u\right)(z) & =\frac{\varepsilon^{-2}}{2 \pi} \int_{0}^{2 \pi}\left[u\left(z+\varepsilon \zeta e^{i \theta}\right)-u(z)\right] d \theta \\
& =\frac{\varepsilon^{-2}}{2 \pi} \int_{0}^{2 \pi}\left\{\frac{1}{2}\left(u\left(z+\varepsilon e^{i \theta} \zeta\right)+u\left(z-\varepsilon e^{i \theta} \zeta\right)\right)-u(z)\right\} d \theta .
\end{aligned}
$$

Since $u$ is plurisubharmonic, and by Proposition 6.7, it follows that $0 \leqq T_{\zeta, \varepsilon} u(z) \leqq$ $A(\eta)$ for $|z| \leqq 1-\eta, \varepsilon \leqq \eta / 2$. Thus by Proposition $6.3, \lim _{\varepsilon \rightarrow 0} T_{\zeta, \varepsilon}(u)=\frac{\partial^{2} u}{\partial \zeta \partial \bar{\zeta}}$ where the convergence is in the weak sense of measures. Now because of the bound for $T_{\zeta, \varepsilon} u$, we then have that $\frac{\partial^{2} u}{\partial \zeta \partial \bar{\zeta}} \in L_{2, \text { loc }}(|z|<1-\eta)$. In particular, the Laplacian of $u$ is locally bounded.

Next let us show that $\frac{\partial^{2} u}{\partial x_{k}^{2}} \in L_{\mathrm{loc}}\left(B^{n}\right)$, where $z_{j}=x_{j}+i x_{n+j}$ are the underlying real coordinates of $\mathbb{C}^{n}$. Since the Laplacian of $u$ is locally bounded, all the second order partial derivatives of $u, \frac{\partial^{2} u}{\partial x_{i} \partial x_{j}}$ are locally in $L^{p}$ for every $p<\infty$. If $e_{j}=$ $(0, \ldots, 0,1,0 \ldots 0)$ is the $j$-th standard basis vector, then

$$
\frac{\varepsilon^{-2}}{2}\left[u\left(z+\varepsilon e_{j}\right)+u\left(z-\varepsilon e_{j}\right)-2 u(z)\right]
$$

converges to $\frac{\partial^{2} u}{\partial x_{j}^{2}}$ in $L, p<\infty$, and thus a subsequence converges almost everywhere. Then again, by Proposition 6.7 , it follows that $\frac{\partial^{2} u}{\partial x_{k}^{2}}(z) \leqq \frac{1}{2} A(\eta)$ for almost
all $|z| \leqq 1-\eta$, and $k=1,2, \ldots, 2 n$.

But, if $\zeta=e_{j}, z_{j}=x_{j}+i x_{n+j}$, we have

$$
0 \leqq \frac{\partial^{2} u}{\partial \zeta \partial \bar{\zeta}}(z)=\frac{1}{4}\left[\frac{\partial^{2} u}{\partial x_{j}^{2}}(z)+\frac{\partial^{2} u}{\partial x_{n+j}^{2}}(z)\right] \leqq A(\eta)
$$


which implies that $\frac{\partial^{2} u}{\partial x_{j}^{2}}, \frac{\partial^{2} u}{\partial x_{n+j}^{2}}$ are also bounded below a.e. on $|z| \leqq 1-\eta$. To see that the mixed derivatives $\frac{\partial^{2} u}{\partial x_{j} \partial x_{k}}$ are bounded, let $X=\frac{\partial}{\partial x_{j}}+\frac{\partial}{\partial x_{k}}$. Then by the above argument $X^{2} u$ is locally bounded, and

$$
\frac{2 \partial^{2} u}{\partial x_{j} \partial x_{k}}=X^{2} u-\frac{\partial^{2} u}{\partial x_{j}^{2}}-\frac{\partial^{2} u}{\partial x_{k}^{2}}
$$

so $u \in L_{2, \text { loc }}^{\infty}\left(B^{n}\right)$, as asserted.

Remark 6.8. Following through the details of the proof of Theorem 6.7, one may obtain the estimate

$$
\left|\frac{\partial^{2} u(z)}{\partial x_{j} \partial x_{k}}\right| \leqq \frac{C}{(1-|z|)^{2}}, \quad|z|<1
$$

on the second derivatives of $u$; where $C$ depends on the derivatives of $\phi$ and $f$ up to second order. The same argument yields uniform estimates for second order tangential derivatives of $u$, if in the proof of Theorem 6.7 the automorphisms $L(z, h)$ are replaced by unitary maps. That is, if $L_{j, k}=\bar{z}_{j} \frac{\partial}{\partial z_{k}}-\bar{z}_{k} \frac{\partial}{\partial z_{j}}$, then

$$
\left|L_{j k} \bar{L}_{p q} u\right| \leqq C
$$

where again $C$ depends on second order derivatives of $\phi$ and $f$. It is possible to refine the argument and improve the estimate (6.12) to

$$
\left|\frac{\partial^{2} u(z)}{\partial x_{j} \partial x_{k}}\right| \leqq \frac{C}{1-|z|} .
$$

It seems reasonable that the factor $(1-|z|)^{-1}$ can be eliminated if $C$ is allowed to depend on derivatives of $\phi$ and $f$ up to fourth order.

\section{Approximations to the Laplacian}

The regularity Theorem 6.9 allows us to reduce the proof of existence of solutions of the Dirichlet problem to functions with locally bounded second derivatives. In this section, we show in Theorem 7.3 that a standard approximation of the Laplacian will essentially allow a further reduction to the $C^{\infty}$ case. In analogy with the operators $T_{\zeta, \varepsilon}$ of Section 6 , an operator $T_{\varepsilon}$ is defined which converges to the Laplacian $\Delta$ as $\varepsilon \rightarrow 0$. The result is much in the same spirit as Littlewood's principle that a measurable function is "almost continuous" and shows that a function $u$ with bounded second partial derivatives behaves "almost everywhere" as though it were of class $C^{2}$. The approximation $T_{\varepsilon}$ and all the arguments are quite standard although somewhat technical. Also, since this is a purely real variable result, we shall work in $\mathbb{R}^{m}$, setting $\mathbb{R}^{2 n}=\mathbb{C}^{n}$ when the Theorem is applied in Section 8.

Let $\Omega$ be a bounded open set in $\mathbb{R}^{m}$. Let $u$ be a locally integrable function on $\Omega$, i.e., $u \in L_{\text {loc }}^{1}(\Omega)$. For $\varepsilon>0$, define a smoothing of $u$ that is different from the 
one used in Section 6,

$$
u_{\varepsilon}(x)=\frac{1}{\tau_{m} \varepsilon^{m}} \int_{|\xi| \leq \varepsilon} u(x+\xi) d V(\xi)
$$
where $d V$ is Lebesgue measure on $\mathbb{R}^{m}$, and $\tau_{m}=\int_{|\xi| \leqq 1} d V(\xi)$ is the volume of the
unit ball in $\mathbb{R}^{m}$. Define

$$
T_{\varepsilon} u=\frac{2(m+2)\left(u_{\varepsilon}-u\right)}{\varepsilon^{2}} .
$$

If $u \in C^{2}(\Omega)$, an easy calculation using the second order Taylor polynomial of $u$ shows that

$$
\lim _{\varepsilon \rightarrow 0} T_{\varepsilon} u(x)=\Delta u(x), \quad u \in C^{2}(\Omega)
$$

and the limit is uniform over compact subsets of $\Omega$. It is well known that the operators $T_{\varepsilon} u$ converge to the Laplacian of $u, \Delta u$, in the distribution sense. For our purposes we need a more refined version of the limit relation (7.2) which applies to functions with bounded Laplacian. The precise version is Theorem 7.3.

We need to use the following fact about the operators $T_{\varepsilon}$.

Proposition 7.1. If $\Delta u \in L_{\mathrm{loc}}^{1}(\Omega)$, then

$\lim _{\varepsilon \rightarrow 0} T_{\varepsilon} u=\Delta u \quad$ in $L_{\text {loc }}^{1}(\Omega)$.

This proposition is well known. We omit the proof.

We also need an estimate on the Lipschitz norm of $T_{\varepsilon} u$ for small $\varepsilon>0$ when $\Delta u \in L_{\mathrm{loc}}^{1}(\Omega)$.

Proposition 7.2. Let $K$ be a compact subset of $\Omega$ of distance greater than $\varepsilon_{0}$ from $\partial \Omega$. There is a constant $C>0$ such that for all $x, y \in K$ and $0<\varepsilon \leqq \varepsilon_{0}$,

$$
\left|T_{\varepsilon} u(x)-T_{\varepsilon} u(y)\right| \leqq C \frac{M|x-y|}{\varepsilon}
$$

where $M=\operatorname{ess} \sup \left\{|\Delta u(\xi)|\right.$ : distance from $\xi$ to $K$ is at most $\left.\varepsilon_{0}\right\}$.

Proof. We shall use the familiar Jensen formula

$$
\frac{1}{\sigma_{m-1}} \int_{|\alpha|=1} u(x+r \alpha) d \sigma(\alpha)=u(x)+\int_{0}^{r} \frac{n(t, x)}{t^{m-1}} d t
$$

where $d \sigma$ is the surface area measure on the unit sphere $S^{m-1}=\left\{\alpha \in R^{m}:|\alpha|=1\right\}$ and $\sigma_{m-1}=\int_{S^{m-1}} d \sigma$ is the area of $S^{m-1}$. The quantity $n(t, x)$ is given by

$$
n(t, x)=\frac{1}{\sigma_{m-1}} \int_{|\xi| \leqq t} \Delta u(x+\xi) d V(\xi)
$$

Combining the Jensen formula with the definition of $T_{\varepsilon}$ gives

$$
\left(T_{\varepsilon} u\right)(x)=\frac{2(m+2) m}{\varepsilon^{m+2}} \int_{0}^{\varepsilon} r^{m-1}\left[\int_{0}^{r} \frac{n(t, x)}{t^{m-1}} d t\right] d r
$$

(since $\sigma_{m-1}=m \tau_{m}$ ). 
We will show an appropriate estimate for $n(t, x)-n(t, y)$. First, note that we always have $|n(\mathrm{t}, x)| \leqq M t^{m}$ so

$$
|n(t, x)-n(t, y)| \leqq 2 M t^{m}, \quad t \leqq \varepsilon_{0} .
$$

If $t \geqq 2|x-y|$, set $E(t)=\left\{\xi \in \mathbb{R}^{m}:|\xi-x| \leqq t\right.$ and $|\xi-y| \geqq t$, or $|\xi-x| \geqq t$ and $\left.|\xi-y| \geqq t\right\}$. Then measure $(E(t)) \leqq C|x-y| t^{m-1}$, since $t \geqq 2|x-y|$. From the formula (7.3) for $n(t, x)$, we see that $|n(t, x)-n(t, y)| \leqq \frac{M}{m-1}[$ measure $(E(t))] \leqq C^{\prime}|x-y| t^{m-1}$,
that is,

$$
|n(t, x)-n(t, y)| \leqq C^{\prime}|x-y| t^{m-1} \quad \text { if } 2|x-y| \leqq t \leqq \varepsilon_{0} .
$$

If $\varepsilon \leqq 2|x-y|, \varepsilon \leqq \varepsilon_{0}$, the estimate of the proposition follows by using (7.5) in formula (7.4). If $2|x-y| \leqq \varepsilon \leqq \varepsilon_{0}$, then again use the formula (7.4), except this time use (7.5) to estimate $n(t, x)-n(t, y)$ when $t \leqq 2|x-y|$ and (7.6) when $t \geqq 2|x-y|$.

We can now prove the exact convergence result we need for the $T_{\varepsilon}$ and $u_{\varepsilon}$.

Theorem 7.3. Suppose $u$ is a function such that $\Delta u \in L_{\mathrm{loc}}^{\infty}(\Omega)$, where $\Omega$ is a bounded open set in $\mathbb{R}^{m}$. Let $\eta>0$ and let $\left\{\varepsilon_{j}\right\}$ be a sequence of positive numbers converging to zero. Then there exists a compact set $F \subset \Omega$ and an integer $j_{0}$ such that

(i) measure $(\Omega \backslash F)<\eta$;

(ii) the restriction of $\Delta u$ to $F$ is (almost everywhere equal to) a continuous function on $F$,

(iii) for all $x \in F$ and $j \geqq j_{0}$, we have $\left|T_{\varepsilon_{j}} u(y)-\Delta u(x)\right| \leqq \eta$ for all $y$ with $|y-x| \leqq \varepsilon_{j}$. Moreover, if all the second partial derivatives of $u, \frac{\partial^{2} u}{\partial x_{i} \partial x_{k}}$, are in $L_{\mathrm{loc}}^{\infty}(\Omega)$,
then we can also choose $F$ so that

(iv) the restriction of $\frac{\partial^{2} u}{\partial x_{i} \partial x_{k}}$ to $F$ is a continuous function; and

(v) for all $x \in F$ and $j \geqq j_{0}$, we have

$$
\left|\frac{\partial^{2} u_{\varepsilon_{j}}}{\partial x_{i} \partial x_{k}}(y)-\frac{\partial^{2} u}{\partial x_{i} \partial x_{k}}(x)\right| \leqq \eta
$$

for all $y$ with $|y-x| \leqq \varepsilon_{j}$.

Proof. Let $\eta_{1}>0$, and let $\left\{\varepsilon_{j}\right\}$ be a sequence of positive numbers, $\varepsilon_{j} \rightarrow 0$. By Egoroff's Theorem ([21], p. 75) and Proposition 7.1, we can find a compact set $F_{1} \subset \Omega$ such that measure $\left[\left(\Omega \backslash F_{1}\right)\right]<\eta$, and

$$
\lim T_{\varepsilon_{j}} u(x)=\Delta u(x) \quad \text { uniformily for } x \in F_{1} .
$$

Note that since all of the $T_{\varepsilon_{j}} u$ are continuous, this limit defines $\Delta u$ as a continuous function on $F_{1}$. If the second partial derivatives $\frac{\partial^{2} u}{\partial x_{i} \partial x_{j}}$ are in $L_{\text {loc }}^{\infty}(\Omega)$ (or just integrable functions, which follows from $\left.\Delta u \in L_{\text {loc }}^{\infty}(\Omega)\right)$, then by Lusin's theorem ([21], p. 56), after possibly shrinking $F_{1}$ slightly, we can assume also that the restriction of each $\frac{\partial^{2} u}{\partial x_{i} \partial x_{j}}$ to $F_{1}$ is a continuous function. 
Now consider the functions

$$
\phi_{s}(x)=\sup _{0<t \leqq s} \frac{\text { measure }\left\{\xi:|\xi-x| \leqq t, \xi \notin F_{1}\right\}}{\tau_{m} t^{m}} .
$$

From the differentiability theory of $L^{1}$ functions, (see e.g. [21]), it follows that $\phi_{s}(x)$ converges almost everywhere on $\Omega$ to the characteristic function of $\Omega \backslash F_{1}$. Since the sequence $\phi_{s}$ is monotone in $s$, it follows that there is a compact subset $F_{2}$ of $F_{1}$ such that measure $\left(\Omega \backslash F_{2}\right)<\eta_{1}$, and a number $t_{0}>0$ such that $\phi_{s}(x)<\eta_{1}$ if $x \in F_{2}$ and $s<t_{0}$. That is,

$$
\text { measure }\{\xi:|\xi-x| \leqq t, \xi \notin F\} \leqq \eta_{1} \tau_{m} t^{m}, \quad x \in F_{2}, \quad 0<t \leqq t_{0},
$$

We set $F=F_{2}$.

The condition (i) holds, if we assume, as we may, that $\eta_{1}<\eta$. Also, we have already verified (ii). To prove (iii), introduce

$$
\omega\left(\varepsilon, F_{1}\right)=\omega(\varepsilon)=\sup \left\{|\Delta u(y)-\Delta u(x)|:|x-y| \leqq 4 \varepsilon, x, y \in F_{1}\right\} .
$$

Since $F_{1}$ is compact and $\Delta u \mid F_{1}$ is continuous, $\omega(\varepsilon) \rightarrow 0$ as $\varepsilon \rightarrow 0$. Suppose $\varepsilon>0$ is such that $4 \varepsilon \leqq t_{0}$. Then if $x \in F=F_{2}$ and $|y-x| \leqq \varepsilon$, there exists $w \in F_{1}$ such that $|w-y| \leqq 2 \eta_{1}^{1 / m} \varepsilon$. For, if not then the entire ball $\left\{\xi:|\xi-y| \leqq 2 \eta_{1}^{1 / m} \varepsilon\right\}$ lies inside $\left\{\xi:|\xi-x| \leqq 2 \varepsilon, \xi \notin F_{2}\right\}$. Then, by (7.8) $\eta_{1} \tau_{m}(2 \varepsilon)^{m} \leqq$ measure $\left\{\xi:|\xi-x| \leqq 2 \varepsilon, \xi \notin F_{2}\right\}<$ $\eta_{1} \tau_{m}(2 \varepsilon)^{m}$, a contradiction. With this choice of $w$, we then have for $x \in F,|y-x| \leqq \varepsilon$

$$
\left|T_{\varepsilon} u(y)-\Delta u(x)\right| \leqq\left|T_{\varepsilon} u(y)-T_{\varepsilon} u(w)\right|+\left|T_{\varepsilon} u(w)-\Delta u(w)\right|+|\Delta u(w)-\Delta u(x)| .
$$

By Proposition 7.2, the first term on the right hand side of (7.9) does not exceed $C M \frac{|y-w|}{\varepsilon} \leqq 2 C M \eta_{1}^{1 / m}$. The last term on the right hand side of (7.9) does not exceed $\omega(\varepsilon)$. And, if $\varepsilon=\varepsilon_{j}$, we have from (7.7) that $\lim \left|T_{\varepsilon_{j}}(w)-\Delta u(w)\right|=0$ uniformly for $w \in F_{1}$. The assertion (iii) therefore follows from (7.9), provided $2 C M \eta_{1}^{1 / m}<\eta$.

The set $F$ has already been chosen so that (iv) holds. To prove (v), write

$$
\frac{\partial^{2} u_{\varepsilon}}{\partial x_{i} \partial x_{k}}(y)-\frac{\partial^{2} u}{\partial x_{i} \partial x_{k}}(x)=\frac{1}{\tau_{m} \varepsilon^{m}} \int_{|\xi| \leqq \varepsilon}\left[\frac{\partial^{2} u}{\partial x_{i} \partial x_{k}}(y+\xi)-\frac{\partial^{2} u}{\partial x_{i} \partial x_{k}}(x)\right] d V(\xi) \text {. }
$$

Set

$$
\tilde{\omega}(\varepsilon)=\operatorname{ess} \sup \left\{\left|\frac{\partial^{2} u}{\partial x_{i} \partial x_{k}}(y)-\frac{\partial^{2} u}{\partial x_{i} \partial x_{k}}(x)\right|:|x-y| \leqq 2 \varepsilon, x, y \in F_{1}, 1 \leqq i, k \leqq m\right\} .
$$

Then if $x \in F \subset F_{1}$ and $y+\xi \in F_{1}$, the integrand in the right hand side of (7.10) does not exceed $\tilde{\omega}(\varepsilon)$. Thus,

$$
\left|\frac{\partial^{2} u_{\varepsilon}}{\partial x_{i} \partial x_{k}}(y)-\frac{\partial^{2} u}{\partial x_{i} \partial x_{k}}(x)\right| \leqq \tilde{\omega}(\varepsilon)+\frac{2 M\left[\text { measure }\left\{\xi: \xi \notin F_{1},|x-\xi| \leqq 2 \varepsilon\right\}\right]}{\tau_{m} \varepsilon^{m}} .
$$

But, $\tilde{\omega}(\varepsilon) \rightarrow 0$ as $\varepsilon \rightarrow 0$, since each function $\frac{\partial^{2} u}{\partial x_{i} \partial x_{k}}$ is continuous on $F_{1}$. Thus, the assertion (v) follows from (7.8) provided $\eta_{1}>0$ is chosen small enough. This completes the proof. 


\section{Existence of Generalized Solutions}

The results of the preceding sections can be combined to show the existence of solutions. The method is to solve progressively more general versions of the problem. The main step is the first step where it is shown that solutions exists on the unit ball in $\mathbb{C}^{n}$ if the data are sufficiently smooth. The reason for starting with the ball is that our regularity Theorem 6.9 can be applied there, and allows us to show that the upper envelope of solutions is actually a generalized solution. We then proceed by making spherical modifications (analogous to the Poisson modifications in the Dirichlet problem for the Laplacian) to solve the problem for more general domains and more general data.

We first treat the following special case.

$$
\begin{aligned}
& \Omega=B^{n}=\text { unit ball in } \mathbb{C}^{n} \\
& d \mu=f d V, \quad f \geqq 0, \quad f^{1 / n} \in C^{2}\left(\overline{B^{n}}\right) \\
& \phi \in C^{2}\left(\partial B^{n}\right) .
\end{aligned}
$$

Under these hypotheses, it follows from Theorem 6.2 (with $\alpha=0$ ) and the regularity Theorem 6.9 that the upper envelope to the classes $\mathscr{B}(\phi, \mu), C \mathscr{B}(\phi, \mu)$, and $\mathscr{F}(\phi, \mu)$ all coincide. Denote this upper envelope by $\boldsymbol{u}$.

Theorem 8.1. Under hypotheses (8.1), we have

$$
\Phi(u)=f^{1 / n} d V,
$$

and

$$
\left(d d^{c} u\right)^{n}=f d V \quad \text { on }|z|<1 .
$$

Thus, $u$ solves the Dirichlet problem (1).

Proof. By Theorem 6.2, $u \in C\left(\overline{B^{n}}\right)$ and $u=\phi$ on $\partial B^{n}$. By Theorem 6.8, the second partial derivatives of $u$ are locally bounded, so from Theorem $5.8, \Phi(u)=g^{1 / n} d V$, $g \geqq 0$, and $\left(d d^{c} u\right)^{n}=g d V$. Thus, it suffices to prove either (8.2) or (8.3). We will prove (8.3).

Now $u$ is the limit of a sequence $u_{j} \in \mathscr{B}(\phi, \mu)$, with $u_{1} \leqq u_{2} \leqq \cdots, u_{j} \rightarrow u$ in $C\left(\overline{B^{n}}\right)$, since $u \in C\left(\overline{B^{n}}\right)$ and $\mathscr{B}(\phi, \mu)$ is closed under finite maxima. Thus, by (4) of Theorem $5.7, u \in \mathscr{B}(\phi, \mu)$. Thus, the locally bounded function $g$ satisfies $g \geqq f$. If $g \equiv f$, we are done, so we may assume that for some small constant $c>0$, the measure of the set

$$
\{z:|z| \leqq 1-c, g(z)>f(z)+c\}
$$

is greater than $c>0$. Set

$$
M=M_{c}=\operatorname{ess} \sup \left\{\left|\frac{\partial^{2} u}{\partial z_{i} \partial \bar{z}_{j}}(z)\right|:|z| \leqq 1-c / 2\right\} .
$$

We have $M<\infty$, by Theorem 6.9 .

Now, choose positive numbers $a, \eta>0$ such that

$$
a<c 4^{-n}\left[2 n !(1+n M)^{n}\right]^{-1}
$$

and

$$
\eta<a[4(2 n+1)]^{-1} .
$$


Let $\left\{\varepsilon_{j}\right\}$ be any sequence of positive numbers tending to zero. Let $F$ be the compact set and $j_{0}$ the positive integer given by Theorem 7.3. We can also assume that $j_{0}$ is so large that

$$
|f(z)-f(w)| \leqq c / 4 \quad \text { if }|z-w| \leqq \varepsilon_{j}, \quad j \geqq j_{0} .
$$

If we write $u_{j}$ for $u_{\varepsilon_{j}}$, then for $z \in F$ and $j \geqq j_{0}$, Theorem 7.3 yields

$$
\left|T_{\varepsilon_{j}} u(w)-\Delta u(z)\right| \varepsilon_{j}^{2} \leqq \eta \varepsilon_{j}^{2}
$$

and

$$
\frac{\partial^{2} u_{j}}{\partial z_{i} \partial \bar{z}_{k}}(w)=\frac{\partial^{2} u}{\partial z_{i} \partial \bar{z}_{k}}(z)+\alpha_{i k}, \quad\left|\alpha_{i k}\right| \leqq \eta
$$

for $z \in F$ and all $w$ with $|w-z| \leqq \varepsilon_{j}$.

Now, because the measure of the complement of $F$ does not exceed $\eta<c / 4$, there exists at least one point $z \in F$ for which $g(z)>f(z)+c$. This point $z$ will be fixed for the remainder of the argument.

Consider the functions

$$
\begin{aligned}
v_{j}(w)= & u_{j}(w)-a|w-z|^{2}+n \eta|w-z|^{2} \\
& -\left[\frac{(\Delta u)(z)}{2(2 n+2)}-a\right] \varepsilon_{j}^{2}-2 n \eta\left(\varepsilon_{j}\right)^{2} .
\end{aligned}
$$

Then for $j \geqq j_{0}$, we assert that $v_{j}$ has the following five properties.

$$
\begin{aligned}
& v_{j} \text { is a } C^{2} \text { function on }|w-z| \leqq \varepsilon_{j} . \\
& v_{j} \text { is plurisubharmonic for }|w-z|<\varepsilon_{j} . \\
& v_{j}(w)<u(w) \quad \text { if }|w-z|=\varepsilon_{j} \\
& \left(d d^{c} v_{j}\right)^{n} \geqq f d V \quad \text { if }|w-z|<\varepsilon_{j} \\
& v_{j}(z)>u(z)
\end{aligned}
$$

If these properties are all verified, then we may find a subsolution larger than $u$ at $z$, namely,

$$
V(w)= \begin{cases}\left.\max \left(v_{j}(w), u(w)\right)\right) & |w-z| \leqq \varepsilon_{j} \\ u(w) & |w-z| \geqq \varepsilon_{j}\end{cases}
$$

Then by (6) of Theorem 5.7, and properties (8.10)-(8.13), $V$ is a subsolution:

$$
V \in C \mathscr{B}(\phi, \mu) \subset \mathscr{B}(\phi, \mu) \text {. }
$$

But, by $(8.14), V(z)>u(z)$ which contradicts the maximality of $u$. The theorem therefore follows from (8.10)-(8.14).

It remains to verify $(8.10)-(8.14)$. Now $(8.10)$ is clear, since $u_{j} \in C^{2}$. To prove (8.11), let I denote the $n \times n$ identity matrix. Then write, using (8.9) and (8.8),

$$
\left[\frac{\partial^{2} v_{j}}{\partial z_{i} \partial \bar{z}_{k}}(w)\right]=\left(\left[\frac{\partial^{2} u}{\partial z_{i} \partial \bar{z}_{k}}(z)\right]-a I\right)+\left(\left[\alpha_{i k}\right]+n \eta I\right) \text {. }
$$


For $|w-z| \leqq \varepsilon_{j}, j \geqq j_{0}$, the inequality of $(8.8)$ shows that $\left[\alpha_{i k}\right]+n \eta I$ is nonnegative. We claim the first matrix on the right hand side of $(8.15)$ is also nonnegative. For, if $0 \leqq \lambda_{1} \leqq \lambda_{2} \leqq \cdots \leqq \lambda_{n}$ are the eigenvalues of $\left[\frac{\partial^{2} u}{\partial z_{i} \partial \bar{z}_{k}}(z)\right]$, then we have $\lambda_{j} \leqq \sum_{1}^{n} \lambda_{j} \leqq n M$ so $\prod_{j=2}^{n} \lambda_{j} \leqq(n M)^{n-1}$. However, $4^{n} n ! \prod_{i=1}^{n} \lambda_{i}=g(z)>f(z)+c \geqq c>0$, so $\lambda_{1} \geqq c 4^{-n}(n !)^{-1}(n M)^{1-n}>a$ by (8.4). Thus, $\left[\frac{\partial^{2} u}{\partial z_{i} \partial \bar{z}_{k}}(z)\right]-a I$ is a positive matrix and (8.11) follows. Note that (8.13) also follows because, from (8.15) we now have

$$
\begin{aligned}
\operatorname{det}\left[\frac{\partial^{2} v_{j}}{\partial z_{i} \partial \bar{z}_{k}}(w)\right] & \geqq \prod_{1}^{n}\left(\lambda_{i}-a\right) \geqq \prod_{1}^{n} \lambda_{i}-a(1+n M)^{n} \\
& =4^{-n}(n !)^{-1} g(z)-a(1+n M)^{n} \\
& \geqq 4^{-n}(n !)^{-1}[g(z)-c / 2] \\
& \geqq 4^{-n}(n !)^{-1}[f(z)+c / 2] .
\end{aligned}
$$

However, from (8.6), $f(z)+c / 2>f(w)$ if $|w-z| \leqq \varepsilon_{j}$, so (8.13) follows.

To prove (8.12) and (8.14), consider the identity,

$$
\begin{aligned}
v_{j}(w)-u(w)= & \frac{1}{2(2 n+2)}\left[T_{\varepsilon_{j}} u(w)-\Delta u(z)\right] \varepsilon_{j}^{2} \\
& +(a-2 n \eta) \varepsilon_{j}^{2}-(a-n \eta)|w-z|^{2} .
\end{aligned}
$$

If we set $|w-z|=\varepsilon_{j}$, then (8.7) yields

$$
v_{j}(w)-u(w) \leqq \frac{\eta \varepsilon_{j}^{2}}{2(2 n+2)}-n \eta \varepsilon_{j}^{2}<0
$$

which proves (8.12). If we set $w=z$, then again, by (8.7),

$$
v_{j}(z)-u(z) \geqq \varepsilon_{j}^{2}\left[a-2 n \eta-\frac{\eta}{2(2 n+2)}\right]>0,
$$

which proves (8.14). This completes the proof of Theorem 8.1.

Next, we will relax the smoothness conditions on $\phi$ and $f$.

Theorem 8.2. Suppose $\Omega=B^{n}$ is the unit ball in $\mathbb{C}^{n}, \phi \in C\left(\partial B^{n}\right)$, and $d \mu=f^{1 / n} d V$ where $f \geqq 0, f \in C\left(\overline{B^{n}}\right)$. Then the upper envelopes of the families $\mathscr{B}(\phi, \mu), C \mathscr{B}(\phi, \mu)$, $\mathscr{F}\left(\phi, \mu^{n}\right)$ coincide. If $u$ is this common upper envelope, then $u \in C\left(\overline{B^{n}}\right)$ and satisfies

$$
\begin{aligned}
& \Phi(u)=f^{1 / n} d V \quad \text { in } B^{n}, \\
& \left(d d^{c} u\right)^{n}=f d V \quad \text { in } B^{n}, \\
& u=\phi \quad \text { in } \partial B^{n} .
\end{aligned}
$$

Proof. Choose a sequence of functions $f_{j}$ with $f_{j}^{1 / n} \in C^{2}\left(\overline{B^{n}}\right)$, and $f_{j}$ decreasing to $f$ uniformly on $\overline{B^{n}}$. Also, choose a sequence of $C^{2}$ functions $\phi_{j}$ on $B^{n}$ such that $\phi_{j}$ increases to $\phi$, uniformly on $\partial B^{n}$. Let $u_{j}$ be the unique plurisubharmonic solution to the Dirichlet problem $\left(d d^{c} u_{j}\right)^{n}=f_{j} d V$ in $B^{n}, u_{j}=\phi_{j}$ in $\partial B^{n}, u_{j} \in C\left(\overline{B^{n}}\right)$. The existence of $u_{j}$ is given by Theorem 8.1, and the uniqueness by Theorem A. Also 
by Theorem $\mathrm{A}$, the sequence $u_{j}$ is increasing. We can choose positive numbers $\eta_{j}$ tending to zero so that $\phi_{j}+\eta_{j} \geqq \phi$ on $\partial B^{n}$. Further, since $\left(\dot{d} d^{c}\left[u_{k}+\varepsilon\left(|z|^{2}-1\right)\right]\right)^{n} \geqq$ $\left(d d^{c} u_{k}\right)^{n}+\varepsilon^{n}\left(d d^{c}|z|^{2}\right)^{n}=f_{k} d V+\varepsilon^{n}\left(d d^{c}|z|^{2}\right)^{n}$, and since $f_{k} \rightarrow f$ uniformly, we can choose positive numbers $\varepsilon_{j} \rightarrow 0$ such that $\left(d d^{c}\left[u_{k}+\varepsilon_{j}\left(|z|^{2}-1\right)\right]^{n} \geqq\left(d d^{c} u_{j}\right)^{n}\right.$ for $k \geqq j$. Therefore, by Theorem $\mathrm{A}$, if $k \geqq j$ we have

$$
u_{k}+\varepsilon_{j}\left(|z|^{2}-1\right) \leqq u_{j}(z)+\eta_{j} \quad \text { for } z \in \overline{B^{n}} \text {. }
$$

Thus, since $u_{j}(z) \leqq u_{k}(z)$, we find that $\lim _{j \rightarrow \infty} u_{j}=u$ exists uniformly on $\overline{B^{n}}$. It follows that $u \in P\left(B^{n}\right)$. Further, $\left(d d^{c} u_{j}\right)^{n} \rightarrow\left(d d^{c} u\right)^{n}$ weakly, by Theorem 2.3, so $\left(d d^{c} u\right)^{n}=f d V$. Further, by (4) of Theorem 5.7, $\Phi(u) \geqq f^{1 / n} d V$, and then by (2) of Theorem 5.8, $\Phi(u)^{n} \leqq\left(d d^{c} u\right)^{n}$ so we have $\Phi(u)=f^{1 / n} d V$.

It then follows from Proposition 6.1 that the upper envelopes of $C \mathscr{B}(\phi, f d V)$ and $\mathscr{F}\left(\phi, f^{n} d V\right)$ coincide with $u$. Further, from Theorem 6.2 (with $\alpha=0$ ), the upper envelopes of $\mathscr{B}(\phi, f d V)$ and $C \mathscr{B}(\phi, f d V)$ coincide. This completes the proof.

We can now prove an existence theorem for more general domains.

Theorem 8.3. Let $\Omega$ be a bounded open set in $\mathbb{C}^{n}$. Let $\phi \in C(\partial \Omega)$ and $d \mu=f d V$ with $f \geqq 0, f \in C(\Omega), d V=$ Lebesgue measure. If

(i) $\mathscr{B}(\phi, \mu)$ is nonempty, and

(ii) the upper envelope $u=\sup \{v: v \in \mathscr{B}(\phi, \mu)\}$ is continuous on $\bar{\Omega}$ with $u=\phi$ on $\partial \Omega$, then $u$ is a solution to the Dirichlet problem

$\left(d d^{c} u\right)^{n}=f d V \quad$ in $\Omega$,

$u \in P(\Omega) \cap C(\bar{\Omega})$,

$u=\phi \quad$ on $\partial \Omega$.

Also, $\Phi(u)=f^{1 / n} d V$.

Proof. We only have to prove that $\left(d d^{c} u\right)^{n}=f d V$ in $\Omega$. To see this, fix $z_{0} \in \Omega$ and choose $\varepsilon>0$ so small that the ball $B\left(z_{0}, \varepsilon\right)=\left\{\left|z-z_{0}\right|<\varepsilon\right\}$ has its closure contained in $\Omega$. By Theorem 8.2, there is a function $v(z)$ on $B\left(z_{0}, \varepsilon\right)$ such that

$$
\begin{aligned}
& v \in P\left(B\left(z_{0}, \varepsilon\right)\right) \cap C\left(\overline{B\left(z_{0}, \varepsilon\right)}\right), \\
& v(z)=u(z) \quad \text { on } \partial B\left(z_{0}, \varepsilon\right), \\
& \Phi(v)=f^{1 / n} d V \quad \text { on } B\left(z_{0}, \varepsilon\right), \\
& \left(d d^{c} v\right)^{n}=f d V \quad \text { on } B\left(z_{0}, \varepsilon\right) .
\end{aligned}
$$

By (2) of Theorem 5.8, we have $\left(d d^{c} v\right)^{n} \leqq\left(d d^{c} u\right)^{n}$ in $B\left(z_{0}, \varepsilon\right)$. Thus, by the minmum principle, Theorem A, we have $v \geqq u$ in $\overline{B\left(z_{0}, \varepsilon\right)}$.

Set $U(z)=v(z)$ if $z \in B\left(z_{0}, \varepsilon\right)$, and $U(z)=u(z)$ if $z \in \bar{\Omega} \backslash B\left(z_{0}, \varepsilon\right)$. Then $U \in C(\bar{\Omega}) \cap P(\Omega)$ and $U=\Phi$ on $\partial \Omega$. We also have $\Phi(U) \geqq f^{1 / n} d V$. Therefore, $U \in \mathscr{B}\left(\phi, f^{1 / n} d V\right)$. Then $U \leqq u$. But, $U \geqq u$ by our construction, so $U \equiv u$. In particular, $u=v$ on $B\left(z_{0}, \varepsilon\right)$, so $\left(d d^{c} u\right)^{n}=f d V$ and $\Phi(u)=f^{1 / n} d V$. This completes the proof.

Finally, we can complete the proof of Theorem D by showing the existence of a solution. 
Proof of Theorem $D$. By Theorem 8.3 , we have to verify that $\mathscr{B}(\phi, \mu)$ is not empty and its upper envelope $u \in C(\vec{\Omega})$, and $u=\phi$ on $\partial \Omega$. When $\Omega$ is strictly pseudoconvex, this is a consequence of the $\alpha=0$ case of Theorem 6.2. Thus,

$$
u=\sup \{v: v \in \mathscr{B}(\phi, \mu)\}
$$

is the solution of the Dirichlet problem (1.1).

\section{The Bremermann Dirichlet Problem and Regularity of Envelopes of Holomorphy}

In [4], Bremermann introduced the family $\mathscr{L}(\phi(z), \Omega)$ of all plurisubharmonic functions $v$ on $\Omega$ such that $\limsup _{z \rightarrow \zeta} v(z) \leqq \phi(\zeta), \zeta \in \partial \Omega$. In our notation, $\mathscr{L}(\phi(z), \Omega)=$ $\mathscr{B}(\phi, 0)$. Bremermann proved that the upper envelope $u$ of $\mathscr{L}$ assumed the boundary values $\phi$ continuously when $\phi \in C(\partial \Omega)$ and $\Omega$ is strictly pseudoconvex. It was later shown by J.B. Walsh [23] that $u \in C(\bar{\Omega})$. It is a consequence of the minimum principle Theorem $\mathrm{A}$ and the existence theorem that $u$ is the unique solution of the Dirichlet problem

$$
\begin{aligned}
& \left(d d^{c} u\right)^{n}=0 \quad \text { in } \Omega, \\
& u=\phi \quad \text { in } \partial \Omega, \\
& u \in C(\bar{\Omega}) \text { and } u \text { plurisubharmonic in } \Omega
\end{aligned}
$$

when $\Omega$ is a strongly pseudoconvex set in $\mathbb{C}^{n}$.

Bremermann also proved ([4], p. 270) that if $K$ is the set of all points $(z, w) \in \mathbb{C}^{n+1}$ such that $z \in \partial \Omega$ and $|w| \leqq e^{-\phi(z)}$ or $z \in \Omega$ and $|w| \leqq e^{-m}, m=\sup \{|\phi(z)|: z \in \partial \Omega\}$, then the envelope of holomorphy of $K$ is

$$
\hat{K}=\left\{(z, w) \in \mathbb{C}^{n+1}:|w| \leqq e^{-u(z)}, z \in \bar{\Omega}\right\} .
$$

Consequently, the regularity results, Theorem $C$ and Theorem 6.2, yield the following regularity of part of the boundary of $\hat{K}$.

Theorem 9.1. If $\Omega=B^{n}$ is the unit ball in $\mathbb{C}^{n}$, and if $\phi \in C^{2}\left(\partial B^{n}\right)$, then $\{(z, w) \in \partial \widehat{K}: z \in \Omega\}$ $=\left\{(z, w) \in \mathbb{C}^{n+1}: z \in \Omega, \log |w|+u(z)=0\right\}$, where $u$ is a plurisubharmonic function on $\Omega$ with locally bounded second partial derivatives. More generally, if $\Omega$ is strictly pseudoconvex with $C^{2}$ boundary, and if $\phi \in \operatorname{Lip}^{\alpha}(\partial \Omega)$, then $u \in \operatorname{Lip}^{\alpha / 2}(\bar{\Omega}), 0 \leqq \alpha \leqq 2$.

\section{References}

1. Alexandrov, A.D.: The Dirichlet problem for the equation Det $\left\|z_{i, j}\right\|=\psi\left(z_{1}, \ldots, z_{n}, x_{1}, \ldots, x_{n}\right)$, I., Vestnik, Leningrad Univ. 13, No. 1, 5-24 (1958)

2. Bergman, S.: Functions of extended class in the theory of functions of several complex variables. Trans. Amer. Math. Soc. 63, 523-547 (1948)

3. Bergman, S.: Kernel functions and extended classes in the theory of functions of complex variables, Colloque sur les fonctions de plusieur variables. Bruxelles pp. 135-157 (1953)

4. Bremermann, H.: On a generalized Dirichlet problem for plurisubharmonic functions and pseudoconvex domains. Characterization of Silov boundaries. Trans. Amer. Math. Soc. 91, 246-276(1959)

5. Cheng, S.-Y., Yau, S.-T.: Complete affine hypersurfaces, Parts I, II, III. To appear

6. Chern, S.S., Levine, Harold I., Nirenberg, L.: Intrinsic norms on a complex manifold. Global Analysis (Papers in honor of K. Kodaira), pp. 119-139 Tokyo: Univ. of Tokyo Press 1969 
7. Chern, S. S., Moser, J.: Real hypersurfaces in complex manifolds. Acta Math. 133, 219-271 (1974)

8. Christoffers, H.: Princeton University Thesis, to appear

9. Diederich, K.: Über die 1. und 2. Ableitungen der Bergmannschen Kernfunktion und ihr Randverhalten. Math. Ann. 203, 129-170 (1963)

10. Fefferman, C.: Monge-Ampere equations, the Bergman Kernel, and geometry of pseudoconvex domains. Ann. of Math., 103, 395-416 (1976)

11. Gluck, H.: Manifolds with preassigned curvature - a survey. Bull. Amer. Math. Soc. 81, 313-329 (1975)

12. Goffman, C., Serrin, J.: Sublinear functions of measures and variational integrals. Duke Math. J. 31, 159-178 (1964)

13. Hörmander, L.: Complex analysis in several variables. New York: North Holland/American Elsevier 1973

14. Hörmander, $L .: L^{2}$ estimates and existence theorems for the $\bar{d}$ operator. Acta Math. 113, 89-152 (1965)

15. Kerzman, N., Kohn, J.J., Nirenberg, L.: Lecture at Amer. Math. Soc. Conference on Several Complex Variables, Williamstown, Mass., August, 1975

16. Koppelman, W.: The Cauchy integral formula for functions of several complex variables, Bull. Amer. Math. Soc. 73, 373-377 (1967)

17. Lelong, P.: Plurisubharmonic functions and positive differential forms. New York: Gordon and Breach 1969

18. Pelles, D. A.: Intrinsic measures on complex manifolds and holomorphic mappings. Amer. Math. Soc. Memoir Number 96, 1970

19. Pogorelov, A. V.: Monge-Ampere equations of elliptic type. Groningen: Noordhoff 1964

20. Pogorelov, A.V.: The Dirichlet problem for the $n$-dimensional analogue of the Monge-Ampere equation, Soviet Math. Dokl. 12, 1727-1731 (1971)

21. Rudin, W.: Real and complex analysis. New York: McGraw Hill 1974

22. Siu, Y. T.: Extension of meromorphic maps. Ann. of Math., 102, 421-462 (1975)

23. Walsh, J. B.: Continuity of envelopes of plurisubharmonic functions. J. Math. Mech. 18, 143-148 (1968) 